\title{
Third-Order Adjoint Sensitivity Analysis of an OECD/NEA Reactor Physics Benchmark: I. Mathematical Framework
}

\author{
Dan Gabriel Cacuci*, Ruixian Fang \\ Center for Nuclear Science and Energy, Department of Mechanical Engineering, University of South Carolina, Columbia, SC, \\ USA \\ Email: *cacuci@cec.sc.edu, fangr@cec.sc.edu
}

How to cite this paper: Cacuci, D.G. and Fang, R.X. (2020) Third-Order Adjoint Sensitivity Analysis of an OECD/NEA Reactor Physics Benchmark: I. Mathematical Framework. American Journal of Computational Mathematics, 10, 503-528.

https://doi.org/10.4236/ajcm.2020.104029

Received: October 13, 2020

Accepted: December 4, 2020

Published: December 7, 2020

Copyright $\odot 2020$ by author(s) and Scientific Research Publishing Inc. This work is licensed under the Creative Commons Attribution International License (CC BY 4.0).

http://creativecommons.org/licenses/by/4.0/

\begin{abstract}
This work extends to third-order previously published work on developing the adjoint sensitivity and uncertainty analysis of the numerical model of a polyethylene-reflected plutonium (acronym: PERP) OECD/NEA reactor physics benchmark. The PERP benchmark comprises 21,976 imprecisely known (uncertain) model parameters. Previous works have used the adjoint sensitivity analysis methodology to compute exactly and efficiently all of the 21,976 first-order and $(21,976)^{2}$ second-order sensitivities of the PERP benchmark's leakage response to all of the benchmark's uncertain parameters, showing that the largest and most consequential $1^{\text {st }}$ - and $2^{\text {nd }}$-order response sensitivities are with respect to the total microscopic cross sections. These results have motivated extending the previous adjoint-based derivations to third-order, leading to the derivation, in this work, of the exact mathematical expressions of the $(180)^{3}$ third-order sensitivities of the PERP leakage response with respect to these total microscopic cross sections. The formulas derived in this work are valid not only for the PERP benchmark but can also be used for computing the $3^{\text {rd }}$-order sensitivities of the leakage response of any nuclear system involving fissionable material and internal or external neutron sources. Subsequent works will use the adjoint-based mathematical expressions obtained in this work to compute exactly and efficiently the numerical values of these $(180)^{3}$ third-order sensitivities (which turned out to be very large and consequential) and use them for a third-order uncertainty analysis of the PERP benchmark's leakage response.
\end{abstract}

\section{Keywords}

Polyethylene-Reflected Plutonium Sphere, $1^{\text {st }}-$ Order, $2^{\text {nd }}$-Order, and $3^{\text {rd }}$-Order Sensitivities, $3^{\text {rd }}$-Order Adjoint Sensitivity Analysis, Microscopic 
Total Cross Sections, Expected Value, Variance and Skewness of Response Distribution

\section{Introduction}

Until recently, only the first-order sensitivities (i.e., functional derivatives) of a computational model's responses (i.e., quantities of interest) to the respective model's imprecisely known (i.e., uncertain) parameters have been taken into account when assessing the uncertainties induced in the respective responses by the parameter uncertainties. The second- and higher-order sensitivities could not be computed, except for very simple models comprising a handful of parameters, so these sensitivities were ignored. The Second-Order Adjoint Sensitivity Analysis Methodology ( $\left.2^{\text {nd }}-A S A M\right)$ recently conceived by Cacuci [1] is the only practical method that enables the exact computation of the large number of $2^{\text {nd }}$-order sensitivities arising in large-scale problems comprising many parameters. The application of the $2^{\text {nd }}-A S A M$ to a multiplying nuclear system with source [2] [3] [4] has opened the way for the large-scale application presented in [5]-[10] to a polyethylene-reflected plutonium (acronym: PERP) OECD/NEA reactor physics benchmark [11]. The numerical model of the PERP benchmark includes 21,976 uncertain parameters, as follows: 180 group-averaged total microscopic cross sections, 21,600 group-averaged scattering microscopic cross sections, 120 fission process parameters, 60 fission spectrum parameters, 10 parameters describing the experiment's nuclear sources, and 6 isotopic number densities.

All of the 21,976 first-order sensitivities and $(21,976)^{2}$ second-order sensitivities of the PERP leakage response with respect to the benchmark's parameters were computed, ranked, and analyzed in [5]-[10]. The results obtained in [5]-[10] showed that the contributions stemming from the second-order sensitivities of the leakage response with respect to the group-averaged microscopic total cross sections are the largest, by a significant margin, by comparison to the contributions from the other uncertain model parameters, including the number of densities, fission parameters, microscopic scattering cross sections, source parameters, etc. For the extreme case of fully correlated microscopic total cross sections, for example, neglecting the $2^{\text {nd }}$-order sensitivities of the leakage response with respect to the total cross sections would cause an error as large as $2000 \%$ in the expected value of the leakage response and up to $6000 \%$ in the variance of the leakage response [5]. Given that the effects of these $2^{\text {nd }}$-order sensitivities are much larger than the effects of the $1^{\text {st }}$-order sensitivities [5] [10], it is logical to posit the question of quantifying the magnitudes and contributions that would stem from the $3^{\text {rd }}$-order sensitivities of the PERP benchmark's total leakage response with respect to the microscopic total cross sections. To enable to compute such $3^{\text {rd }}$-order sensitivities, Cacuci [12] has recently conceived the "third-order adjoint sensitivity analysis methodology for reaction rate responses 
in a multiplying nuclear system with source." Cacuci's results [12] are applied in this work to the PERP benchmark in order to derive the exact analytical expressions of the $3^{\text {rd }}$-order sensitivities of the PERP benchmark's leakage response with respect to this benchmark's microscopic total cross sections. Two subsequent works, designated as Part II [13] and Part III [14], respectively, will report numerical results as follows: 1) Part II [13] will present the numerical values the $3^{\text {rd }}$-order sensitivities derived in the present work, showing that the largest of these is over 400 times larger than the largest 2 nd-order sensitivity and is over 20,000 times larger than the largest 1st-order sensitivity; and 2) Part III [14] will quantify the effects of the $3^{\text {rd }}$-order sensitivities on the expected values, the standard deviation and the skewness of the PERP's leakage response, and will compare these effects to those produced by the $1^{\text {st }}$-order and, respectively, $2^{\text {nd }}$-order sensitivities.

This work is organized as follows: Section 2 describes the methodology for computing the leakage response of the PERP benchmark. Section 3 presents the derivation of the exact analytical expressions of the third-order mixed sensitivities of the PERP leakage response to total cross sections. Section 4 concludes this work.

\section{Mathematical Methodology for Computing the Leakage Response of the Polyethylene-Reflected Plutonium (PERP) Metal Sphere Benchmark}

This section presents the derivation of the exact analytical expressions of the $3^{\text {rd }}$-order sensitivities of the polyethylene-reflected plutonium (acronym: PERP) metal sphere OECD/NEA benchmark [11] total leakage response with respect to this benchmark's total cross sections, thus continuing the work presented in [5]-[10]. The numerical modeling of the PERP benchmark has been already described in [5]-[10] but, for convenient reference, the materials of the PERP spherical core and spherical-shell reflector, respectively, are specified in Table A1 in the Appendix. As in [5]-[10], the multigroup discrete ordinates particle transport code PARTISN [15] together with neutron sources computed using the code SOURCES4C [16] have been employed to perform the numerical computations of the various quantities to be derived in this section. The multigroup neutron fluxes computed by PARTISN are the solutions of the following multigroup neutron transport equation with a spontaneous fission source:

$$
\begin{gathered}
B^{g}(\boldsymbol{\alpha}) \varphi^{g}(r, \boldsymbol{\Omega})=Q^{g}(r), \quad g=1, \cdots, G, \\
\varphi^{g}\left(r_{d}, \boldsymbol{\Omega}\right)=0, \boldsymbol{\Omega} \cdot \boldsymbol{n}<0, \quad g=1, \cdots, G,
\end{gathered}
$$

where $r_{d}$ is the radius of the PERP sphere, and where

$$
\begin{aligned}
& B^{g}(\boldsymbol{\alpha}) \varphi^{g}(r, \boldsymbol{\Omega}) \triangleq \boldsymbol{\Omega} \cdot \nabla \varphi^{g}(r, \boldsymbol{\Omega})+\Sigma_{t}^{g}(\boldsymbol{\alpha} ; r) \varphi^{g}(r, \boldsymbol{\Omega}) \\
& -\sum_{g^{\prime}=1}^{G} \int_{4 \pi} \mathrm{d} \boldsymbol{\Omega}^{\prime} \varphi^{g^{\prime}}\left(r, \boldsymbol{\Omega}^{\prime}\right)\left[\Sigma_{s}^{g^{\prime} \rightarrow g}\left(\boldsymbol{\alpha} ; r, \boldsymbol{\Omega}^{\prime} \rightarrow \boldsymbol{\Omega}\right)+\chi^{g}(\boldsymbol{\alpha} ; r)(v \Sigma)_{f}^{g^{\prime}}(\boldsymbol{\alpha} ; r)\right],
\end{aligned}
$$




$$
Q^{g}(r) \triangleq \sum_{k=1}^{N_{f}} \lambda_{k} N_{k, 1} F_{k}^{S F} v_{k}^{S F}\left(\frac{2}{\sqrt{\pi a_{k}^{3} b_{k}}} \mathrm{e}^{-\frac{a_{k} b_{k}}{4}}\right) \int_{E^{g+1}}^{E^{g}} \mathrm{~d} E \mathrm{e}^{-E / a_{k}} \sinh \sqrt{b_{k} E} .
$$

The Boltzmann-operator $B^{g}(\boldsymbol{\alpha})$ in Equation (3) contains implicitly a factor $1 / 4 \pi$ in its scattering and fission terms, to conform to the convention used by PARTISN [15]. As in [5]-[10], the PARTISN [15] computations used the MENDF71X [17] 618-group cross sections collapsed to 30 energy-groups, as shown in Table A2 in the Appendix, in conjunction with a $P_{3}$-Legendre expansion of the scattering cross section, an angular quadrature of $S_{256}$, and a fine-mesh spacing of $0.005 \mathrm{~cm}$ (comprising 759 meshes for the plutonium sphere of radius of $3.794 \mathrm{~cm}$, and 762 meshes for the polyethylene shell of thickness of $3.81 \mathrm{~cm}$ ). The symbols used in Equations (1) through (4) have their usual meanings and are summarized, for convenient referencing, in the Appendix.

The mathematical expression of the PERP benchmark's leakage response, denoted as $L(\boldsymbol{\alpha})$, is provided below:

$$
L(\boldsymbol{\alpha}) \triangleq \int_{S_{b}} \mathrm{~d} S \sum_{g=1}^{G} \int_{\boldsymbol{\Omega} \cdot \boldsymbol{n}>0} \mathrm{~d} \boldsymbol{\Omega} \boldsymbol{\Omega} \cdot \boldsymbol{n} \varphi^{g}(r, \boldsymbol{\Omega}) .
$$

The vector $\alpha$, which appears in the expressions of the Boltzmann-operator $B^{g}(\boldsymbol{\alpha})$ and the leakage response $L(\boldsymbol{\alpha})$, in Equation (3) and Equation (5), respectively, represents the "vector of imprecisely known model parameters", and is defined as follows:

$$
\begin{aligned}
& \boldsymbol{\alpha} \triangleq\left[\alpha_{1}, \cdots, \alpha_{J_{\alpha}}\right]^{\dagger} \triangleq\left[\boldsymbol{\sigma}_{t} ; \boldsymbol{\sigma}_{s} ; \boldsymbol{\sigma}_{f} ; \boldsymbol{v} ; \boldsymbol{p} ; \boldsymbol{q} ; \boldsymbol{N}\right]^{\dagger}, \\
& J_{\alpha}=J_{\sigma t}+J_{\sigma s}+J_{\sigma f}+J_{v}+J_{p}+J_{q}+J_{n} .
\end{aligned}
$$

The components of the vector $\alpha$ are described in the Appendix. Since only the effects of the uncertainties in the total macroscopic cross sections will be considered in this work, only the components of the vector $t$ for the parameters of macroscopic total cross sections will be explicitly used; they are reproduced below from the Appendix:

$$
\boldsymbol{t} \triangleq\left[t_{1}, \cdots, t_{J_{t}}\right]^{\dagger} \triangleq\left[t_{1}, \cdots, t_{J_{\sigma t}} ; n_{1}, \cdots, n_{J_{n}}\right]^{\dagger} \triangleq\left[\sigma_{t} ; \boldsymbol{N}\right]^{\dagger}, J_{t}=J_{\sigma t}+J_{n},
$$

where

$$
\begin{gathered}
\sigma_{t} \triangleq\left[t_{1}, \cdots, t_{J_{\sigma t}}\right]^{\dagger} \triangleq\left[\sigma_{t, i=1}^{1}, \sigma_{t, i=1}^{2}, \cdots, \sigma_{t, i=1}^{G}, \cdots, \sigma_{t, i}^{g}, \cdots, \sigma_{t, i=I}^{1}, \cdots, \sigma_{t, i=I}^{G}\right]^{\dagger}, \\
i=1, \cdots, I=6 ; g=1, \cdots, G=30 ; J_{\sigma t}=I \times G \\
\quad N \triangleq\left[n_{1}, \cdots, n_{J_{n}}\right]^{\dagger} \triangleq\left[N_{1,1}, N_{2,1}, N_{3,1}, N_{4,1}, N_{5,2}, N_{6,2}\right]^{\dagger}, J_{n}=6 .
\end{gathered}
$$

In Equations (7) through (9), the dagger denotes "transposition," $\sigma_{t, i}^{g}$ denotes the microscopic total cross section for isotope $i$ and energy group $g$, $N_{i, m}$ denotes the respective isotopic number density for isotope $i$ and material $m, J_{\sigma t}$ denotes the total number of microscopic total cross sections, and $J_{n}$ denotes the total number of isotopic number densities in the model. Thus, the vector $t$ comprises a total of $J_{t}=J_{\sigma t}+J_{n}=30 \times 6+6=186$ uncertain model parameters. However, only the components 
$\sigma_{t} \triangleq\left[t_{1}, \cdots, t_{J_{\sigma t}}\right]^{\dagger} \triangleq\left[\sigma_{t, i=1}^{1}, \sigma_{t, i=1}^{2}, \cdots, \sigma_{t, i=1}^{G}, \cdots, \sigma_{t, i}^{g}, \cdots, \sigma_{t, i=I}^{1}, \cdots, \sigma_{t, i=I}^{G}\right]^{\dagger}$,

$i=1, \cdots, I=6, g=1, \cdots, G=30, J_{\sigma t}=I \times G=180$, will be considered in this work. Thus, the numbers of sensitivities of the PERP leakage response with respect to the total microscopic cross sections are as follows: 1) 180 first-order sensitivities; 2) 32,400 second-order sensitivities, of which 16,290 are distinct; and 3) 5,832,000 third-order sensitivities, of which 988,260 are distinct. The 180 first-order and 16,290 second-order sensitivities were obtained and analyzed in [5]. The exact mathematical expressions of the 988,260 distinct third-order sensitivities will be obtained in Section 3 of this work, and their numerical values and numerical effects on the variance and skewness of the PERP leakage response will be presented in the accompanying Part II [13] and Part III [14].

\section{Exact Analytical Expressions of the Third-Order Mixed Sensitivities of the PERP Leakage Response to Total Cross Sections}

This Section will present the derivation of the $3^{\text {rd }}$-order mixed sensitivities $\partial^{3} L(\alpha) / \partial t_{j} \partial t_{k} \partial t_{\ell}, j, k, \ell=1, \cdots, J_{\sigma t}$ of the PERP leakage response with respect to the group-averaged microscopic total cross sections. These $3^{\text {rd }}$-order sensitivities will be derived by using two alternative procedures, which will be presented in sections 3.1 and 3.2, respectively. In section 3.1, the sensitivities $\partial^{3} L(\alpha) / \partial t_{j} \partial t_{k} \partial t_{\ell}, j, k, \ell=1, \cdots, J_{\sigma t}$ will be obtained as a particular case of the general expressions derived in [12]. In section 3.2, the expressions of $\partial^{3} L(\alpha) / \partial t_{j} \partial t_{k} \partial t_{\ell}, j, k, \ell=1, \cdots, J_{\sigma t}$ by applying the concepts presented in [12] to the expression of the $2^{\text {nd }}$-order sensitivities $\partial^{2} L(\alpha) / \partial t_{j} \partial t_{k}, j, k=1, \cdots, J_{\sigma t}$, which were derived in Ref. [5]. It will be shown that these two alternative procedures will yield identical expressions for the corresponding $3^{\text {rd }}$-order sensitivities, as would be expected.

\subsection{Computing $\partial^{3} L(\alpha) / \partial t_{j} \partial t_{k} \partial t_{\ell}, j, k, \ell=1, \cdots, J_{\sigma t}$ by Particularizing the General Expressions Obtained in Ref. [12] for the PERP Leakage Response Sensitivities to Total Cross Sections}

The general expression of the $3^{\text {rd }}$-order sensitivities of a reaction-rate type response to the nuclear data characterizing a physical system modeled by the multigroup neutron transport equation has been derived in the Appendix of [12]. This general expression can be specialized for the PERP benchmark's leakage response and total microscopic cross sections by introducing the following correspondences:

$$
\begin{gathered}
\Sigma_{d}^{g}(\alpha) \triangleq \Omega \cdot \boldsymbol{n} \delta\left(r-r_{d}\right), \\
\alpha_{i_{1}} \rightarrow t_{j} \equiv \sigma_{t, i_{j}}^{g_{j}} ; i_{1} \rightarrow j=1, \cdots, J_{\sigma t} ; \\
\alpha_{i_{2}} \rightarrow t_{k} \equiv \sigma_{t, i_{k}}^{g_{k}} ; i_{2} \rightarrow k=1, \cdots, J_{\sigma t} ; \\
\alpha_{i_{3}} \rightarrow t_{\ell} \equiv \sigma_{t, i_{\ell}}^{g_{\ell}} ; i_{3} \rightarrow \ell=1, \cdots, J_{\sigma t} .
\end{gathered}
$$


It has been shown in [5] and it can also be observed directly that the following relations hold when $t_{j} \equiv \sigma_{t, i_{j}}^{g_{j}}$ and $t_{k} \equiv \sigma_{t, i_{k}}^{g_{k}}$, respectively:

$$
\begin{gathered}
\frac{\partial \Sigma_{t}^{g}(\boldsymbol{t})}{\partial t_{j}} \triangleq \frac{\partial \Sigma_{t}^{g}(\boldsymbol{t})}{\partial \sigma_{t, i_{j}}^{g_{j}}}=\frac{\partial\left(\sum_{m=1}^{M} \sum_{i=1}^{I} N_{i, m} \sigma_{t, i}^{g}\right)}{\partial \sigma_{t, i_{j}}^{g_{j}}}=\delta_{g_{j} g} N_{i_{j}, m_{j}}, \\
\frac{\partial^{2} \Sigma_{t}^{g}(\boldsymbol{t})}{\partial t_{j} \partial t_{k}} \triangleq \frac{\partial^{2} \Sigma_{t}^{g}(\boldsymbol{t})}{\partial \sigma_{t, i_{j}}^{g_{j}} \partial \sigma_{t, i_{k}}^{g_{k}}}=\frac{\partial\left(\delta_{g_{j} g} N_{i_{j}, m_{j}}\right)}{\partial \sigma_{t, i_{k}}^{g_{k}}}=0,
\end{gathered}
$$

where the subscripts $i_{j}, g_{j}$ and $m_{j}$ denote the isotope, the energy group and material associated with the parameter $\sigma_{t, i_{j}}^{g_{j}}$, respectively; the subscripts $i_{k}, g_{k}$ and $m_{k}$ denote the isotope, the energy group and material associated with the parameter $\sigma_{t, i_{k}}^{g_{k}}$, respectively; and $\delta_{g_{j} g}$ denotes the Kronecker-delta functional (e.g., $\delta_{g_{j} g}=1$ if $g_{j}=g ; \delta_{g_{j} g}=0$ if $g_{j} \neq g$ ).

It has been shown in the Appendix of [12] that the multigroup $3^{\text {rd }}$-order sensitivities of a reaction-rate type response to the parameters in a physical system modeled by the multigroup neutron transport equation are generally given by the following expression:

$$
\begin{aligned}
& \frac{\partial^{3} R(\boldsymbol{\alpha}, \varphi)}{\partial \alpha_{i_{3}} \partial \alpha_{i_{2}} \partial \alpha_{i_{1}}} \\
& =\left\langle\varphi^{g}(\boldsymbol{r}, \boldsymbol{\Omega}), \frac{\partial^{3} \Sigma_{d}^{g}(\boldsymbol{\alpha})}{\partial \alpha_{i_{3}} \partial \alpha_{i_{2}} \partial \alpha_{i_{1}}}\right\rangle_{(1)}+\left\langle\psi^{(1), g}(\boldsymbol{r}, \boldsymbol{\Omega}), \frac{\partial^{3} Q^{(1), g}(\boldsymbol{\alpha})}{\partial \alpha_{i_{3}} \partial \alpha_{i_{2}} \partial \alpha_{i_{1}}}\right\rangle_{(1)} \\
& +\left\langle\psi_{1}^{(2), g}\left(i_{1} ; \boldsymbol{r}, \boldsymbol{\Omega}\right), \frac{\partial^{2} Q^{(1), g}(\boldsymbol{\alpha})}{\partial \alpha_{i_{3}} \partial \alpha_{i_{2}}}\right\rangle_{(1)}+\left\langle\psi_{2}^{(2), g}\left(i_{1} ; \boldsymbol{r}, \boldsymbol{\Omega}\right), \frac{\partial^{2} Q^{(2), g}(\boldsymbol{\alpha})}{\partial \alpha_{i_{3}} \partial \alpha_{i_{2}}}\right\rangle_{(1)} \\
& +\left\langle\psi_{1}^{(3), g}\left(i_{1}, i_{2} ; \boldsymbol{r}, \boldsymbol{\Omega}\right), \frac{\partial Q^{(1), g}(\boldsymbol{\alpha})}{\partial \alpha_{i_{3}}}\right\rangle_{(1)}+\left\langle\psi_{2}^{(3), g}\left(i_{1}, i_{2} ; \boldsymbol{r}, \boldsymbol{\Omega}\right), \frac{\partial Q^{(2), g}(\boldsymbol{\alpha})}{\partial \alpha_{i_{3}}}\right\rangle_{(1)} \\
& +\left\langle\psi_{3}^{(3), g}\left(i_{1}, i_{2} ; \boldsymbol{r}, \boldsymbol{\Omega}\right), \frac{\partial Q_{1}^{(3), g}(\boldsymbol{\alpha})}{\partial \alpha_{i_{3}}}\right\rangle_{(1)}+\left\langle\psi_{4}^{(3), g}\left(i_{1}, i_{2} ; \boldsymbol{r}, \boldsymbol{\Omega}\right), \frac{\partial Q_{2}^{(3), g}(\boldsymbol{\alpha})}{\partial \alpha_{i_{3}}}\right\rangle_{(1)}, \\
& \quad \text { for } i_{1}=1, \cdots, J_{\alpha} ; i_{2}=1, \cdots, i_{1} ; i_{3}=1, \cdots, i_{2},
\end{aligned}
$$

where the inner product $\langle\boldsymbol{u}(\boldsymbol{r}, \boldsymbol{\Omega}), \boldsymbol{w}(\boldsymbol{r}, \boldsymbol{\Omega})\rangle_{(1)}$ between two vector-values functions, each having $G$ components of the form

$\boldsymbol{u}(\boldsymbol{r}, \boldsymbol{\Omega}) \triangleq\left[u^{1}(\boldsymbol{r}, \boldsymbol{\Omega}), \cdots, u^{g}(\boldsymbol{r}, \boldsymbol{\Omega}), \cdots, u^{G}(\boldsymbol{r}, \boldsymbol{\Omega})\right]^{\dagger}$ and

$\boldsymbol{w}(\boldsymbol{r}, \boldsymbol{\Omega}) \triangleq\left[w^{1}(\boldsymbol{r}, \boldsymbol{\Omega}), \cdots, w^{g}(\boldsymbol{r}, \boldsymbol{\Omega}), \cdots, w^{G}(\boldsymbol{r}, \boldsymbol{\Omega})\right]^{\dagger}$, is defined as follows:

$$
\left\langle u^{g}(\boldsymbol{r}, \boldsymbol{\Omega}), w^{g}(\boldsymbol{r}, \boldsymbol{\Omega})\right\rangle_{(1)} \triangleq \sum_{g=1}^{G} \int \mathrm{d} V \int_{4 \pi} \mathrm{d} \boldsymbol{\Omega} u^{g}(\boldsymbol{r}, \boldsymbol{\Omega}) w^{g}(\boldsymbol{r}, \boldsymbol{\Omega}) .
$$

In Equation (14), the $3^{\text {rd }}$-level adjoint functions $\psi_{j}^{(3), g}\left(i_{1}, i_{2} ; \boldsymbol{r}, \boldsymbol{\Omega}\right), j=1,2,3,4 ; g=1, \cdots, G$ are the solutions of the following multigroup form of the "third-level adjoint sensitivity system" ( $3^{\text {rd }}-$ LASS) presented in the Appendix of [12]:

$$
A^{g}(\boldsymbol{\alpha}) \psi_{4}^{(3), g}\left(i_{1}, i_{2} ; \boldsymbol{r}, \boldsymbol{\Omega}\right)=\nabla_{\psi_{2}^{(2)}\left(i_{1}\right)} R^{(2), g}(\boldsymbol{\alpha}),
$$




$$
\begin{gathered}
\psi_{4}^{(3), g}\left(i_{1}, i_{2} ; \boldsymbol{r}_{d}, \boldsymbol{\Omega}\right)=0, \boldsymbol{\Omega} \cdot \boldsymbol{n}>0, i_{1}=1, \cdots, J_{\alpha} ; i_{2}=1, \cdots, i_{1} ; \\
A^{g}(\boldsymbol{\alpha}) \psi_{1}^{(3), g}\left(i_{1}, i_{2} ; \boldsymbol{r}, \boldsymbol{\Omega}\right)=\nabla_{\varphi} R^{(2), g}(\boldsymbol{\alpha})-\left[F_{41}^{(3), g}\right]^{*} \psi_{4}^{(3), g}\left(i_{1}, i_{2} ; \boldsymbol{r}, \boldsymbol{\Omega}\right), \\
\psi_{1}^{(3), g}\left(i_{1}, i_{2} ; \boldsymbol{r}_{d}, \boldsymbol{\Omega}, E\right)=0, \boldsymbol{\Omega} \cdot \boldsymbol{n}>0, i_{1}=1, \cdots, J_{\alpha} ; i_{2}=1, \cdots, i_{1} ; \\
B^{g}(\boldsymbol{\alpha}) \psi_{3}^{(3), g}\left(i_{1}, i_{2} ; \boldsymbol{r}, \boldsymbol{\Omega}\right)=\nabla_{\psi_{1}^{(2)}\left(i_{1}\right)} R^{(2), g}(\boldsymbol{\alpha}), \\
\psi_{3}^{(3), g}\left(i_{1}, i_{2} ; \boldsymbol{r}_{d}, \boldsymbol{\Omega}\right)=0, \boldsymbol{\Omega} \cdot \boldsymbol{n}<0, i_{1}=1, \cdots, J_{\alpha} ; i_{2}=1, \cdots, i_{1} ; \\
B^{g}(\boldsymbol{\alpha}) \psi_{2}^{(3), g}\left(i_{1}, i_{2} ; \boldsymbol{r}, \boldsymbol{\Omega}\right)=\nabla_{\psi^{(1)}} R^{(2), g}(\boldsymbol{\alpha})-\left[F_{32}^{(3), g}\right]^{*} \psi_{3}^{(3), g}\left(i_{1}, i_{2} ; \boldsymbol{r}, \boldsymbol{\Omega}\right), \\
\psi_{2}^{(3), g}\left(i_{1}, i_{2} ; \boldsymbol{r}_{d}, \boldsymbol{\Omega}\right)=0, \boldsymbol{\Omega} \cdot \boldsymbol{n}<0, i_{1}=1, \cdots, J_{\alpha} ; i_{2}=1, \cdots, i_{1},
\end{gathered}
$$

where

$$
\begin{aligned}
& A^{g}(\boldsymbol{\alpha}) \psi_{1}^{(3), g}\left(i_{1}, i_{2} ; \boldsymbol{r}, \boldsymbol{\Omega}\right) \\
& \triangleq-\boldsymbol{\Omega} \cdot \nabla \psi_{1}^{(3), g}\left(i_{1}, i_{2} ; \boldsymbol{r}, \boldsymbol{\Omega}\right)+\Sigma_{t}^{g}(\boldsymbol{t}) \psi_{1}^{(3), g}\left(i_{1}, i_{2} ; \boldsymbol{r}, \boldsymbol{\Omega}\right) \\
& \quad-\sum_{g^{\prime}=1}^{G} \int_{4 \pi} \mathrm{d} \boldsymbol{\Omega}^{\prime} \psi_{1}^{(3), g^{\prime}}\left(i_{1}, i_{2} ; \boldsymbol{r}, \boldsymbol{\Omega}\right)\left[\Sigma_{s}^{g \rightarrow g^{\prime}}\left(\boldsymbol{s} ; \boldsymbol{\Omega} \rightarrow \boldsymbol{\Omega}^{\prime}\right)+\chi^{g^{\prime}}(\boldsymbol{p}) v \Sigma_{f}^{g}(\boldsymbol{f})\right],
\end{aligned}
$$

and where the various quantities serving as "sources" on the right-sides of the $3^{\text {rd }}$-LASS are defined as follows:

$$
\begin{aligned}
& \frac{\partial Q^{(1), g}(\boldsymbol{\alpha} ; \varphi)}{\partial \alpha_{i_{2}}} \triangleq \frac{\partial Q^{g}(\boldsymbol{\alpha} ; \boldsymbol{r}, \boldsymbol{\Omega})}{\partial \alpha_{i_{2}}}-\frac{\partial\left[B^{g}(\boldsymbol{\alpha}) \varphi^{g}(\boldsymbol{r}, \boldsymbol{\Omega})\right]}{\partial \alpha_{i_{2}}}, \\
& \frac{\partial^{2} Q^{(1), g}(\boldsymbol{\alpha} ; \varphi)}{\partial \alpha_{i_{3}} \partial \alpha_{i_{2}}} \triangleq \frac{\partial^{2} Q^{g}(\boldsymbol{\alpha} ; \boldsymbol{r}, \boldsymbol{\Omega})}{\partial \alpha_{i_{3}} \partial \alpha_{i_{2}}}-\frac{\partial^{2}\left[B^{g}(\boldsymbol{\alpha}) \varphi^{g}(\boldsymbol{r}, \boldsymbol{\Omega})\right]}{\partial \alpha_{i_{3}} \partial \alpha_{i_{2}}}, \\
& \frac{\partial Q^{(2), g}\left(\boldsymbol{\alpha} ; \psi^{(1)}\right)}{\partial \alpha_{i_{2}}}=\frac{\partial \Sigma_{d}^{g}(\boldsymbol{\alpha} ; \boldsymbol{r}, \boldsymbol{\Omega})}{\partial \alpha_{i_{2}}}-\frac{\partial\left[A^{g}(\boldsymbol{\alpha}) \psi^{(1), g}(\boldsymbol{r}, \boldsymbol{\Omega})\right]}{\partial \alpha_{i_{2}}}, \\
& \nabla_{\psi_{2}^{(2)}\left(i_{1}\right)} R^{(2), g}(\alpha) \triangleq \frac{\partial Q^{(2), g}(\alpha)}{\partial \alpha_{i_{2}}}, \\
& {\left[F_{41}^{(3), g}\right]^{*} \psi_{4}^{(3), g}\left(i_{1}, i_{2} ; \boldsymbol{r}, \boldsymbol{\Omega}\right)} \\
& \triangleq \psi_{4}^{(3), g}\left(i_{1}, i_{2} ; \boldsymbol{r}, \boldsymbol{\Omega}\right) \frac{\partial \Sigma_{t}^{g}(\boldsymbol{t})}{\partial \alpha_{i_{1}}}-\sum_{g^{\prime}=1}^{G} \int_{4 \pi} \mathrm{d} \boldsymbol{\Omega}^{\prime} \psi_{4}^{(3), g^{\prime}}\left(i_{1}, i_{2} ; \boldsymbol{r}, \boldsymbol{\Omega}^{\prime}\right) \\
& \times\left\{\frac{\partial \Sigma_{s}^{g \rightarrow g^{\prime}}\left(s ; \boldsymbol{\Omega} \rightarrow \boldsymbol{\Omega}^{\prime}\right)}{\partial \alpha_{i_{1}}}+\frac{\partial\left[v \Sigma_{f}^{g}(\boldsymbol{f})\right]}{\partial \alpha_{i_{1}}} \chi^{g^{\prime}}+v \Sigma_{f}^{g}(\boldsymbol{f}) \frac{\partial \chi^{g^{\prime}}}{\partial \alpha_{i_{1}}}\right\}, \\
& \nabla_{\psi_{1}^{(2)}\left(i_{1}\right)} R^{(2), g}(\alpha) \triangleq \frac{\partial Q^{(1), g}(\alpha, \varphi)}{\partial \alpha_{i_{2}}}, \\
& \nabla_{\varphi} R^{(2), g}(\alpha) \\
& \triangleq \frac{\partial^{2} \Sigma_{d}^{g}(\boldsymbol{\alpha} ; \boldsymbol{r}, \boldsymbol{\Omega})}{\partial \alpha_{i_{1}} \partial \alpha_{i_{2}}}-\psi_{1}^{(2), g}\left(i_{1} ; \boldsymbol{r}, \boldsymbol{\Omega}\right) \frac{\partial \Sigma_{t}^{g}(\boldsymbol{t})}{\partial \alpha_{i_{2}}}+\sum_{g^{\prime}=1}^{G} \int_{4 \pi} \mathrm{d} \boldsymbol{\Omega}^{\prime} \psi_{1}^{(2), g^{\prime}}\left(i_{1} ; \boldsymbol{r}, \boldsymbol{\Omega}^{\prime}\right)
\end{aligned}
$$




$$
\begin{aligned}
& \times\left\{\frac{\partial \Sigma_{s}^{g \rightarrow g^{\prime}}\left(\boldsymbol{s} ; \boldsymbol{\Omega} \rightarrow \boldsymbol{\Omega}^{\prime}\right)}{\partial \alpha_{i_{2}}}+v \Sigma_{f}^{g}(\boldsymbol{f}) \frac{\partial \chi^{g^{\prime}}}{\partial \alpha_{i_{2}}}+\frac{\partial\left[v \Sigma_{f}^{g}(\boldsymbol{f})\right]}{\partial \alpha_{i_{2}}} \chi^{g^{\prime}}\right\} \\
& -\psi^{(1), g}(\boldsymbol{r}, \boldsymbol{\Omega}) \frac{\partial^{2} \Sigma_{t}^{g}(\boldsymbol{t})}{\partial \alpha_{i_{1}} \partial \alpha_{i_{2}}}+\sum_{g^{\prime}=1}^{G} \int_{4 \pi} \mathrm{d} \boldsymbol{\Omega}^{\prime} \psi^{(1), g^{\prime}}\left(\boldsymbol{r}, \boldsymbol{\Omega}^{\prime}\right) \\
& \times\left\{\frac{\partial^{2} \Sigma_{s}^{g \rightarrow g^{\prime}}\left(\boldsymbol{s} ; \boldsymbol{\Omega} \rightarrow \boldsymbol{\Omega}^{\prime}\right)}{\partial \alpha_{i_{1}} \partial \alpha_{i_{2}}}+\chi^{g^{\prime}} \frac{\partial^{2}\left[v \Sigma_{f}^{g}(\boldsymbol{f})\right]}{\partial \alpha_{i_{1}} \partial \alpha_{i_{2}}}+\frac{\partial\left[v \Sigma_{f}^{g}(\boldsymbol{f})\right]}{\partial \alpha_{i_{1}}} \frac{\partial \chi^{g^{\prime}}}{\partial \alpha_{i_{2}}}\right. \\
& \left.+\frac{\partial \chi^{g^{\prime}}}{\partial \alpha_{i_{1}}} \frac{\partial\left[v \Sigma_{f}^{g}(\boldsymbol{f})\right]}{\partial \alpha_{i_{2}}}+v \Sigma_{f}^{g}(\boldsymbol{f}) \frac{\partial^{2} \chi^{g^{\prime}}}{\partial \alpha_{i_{1}} \partial \alpha_{i_{2}}}\right\} \\
& \nabla_{\psi^{(1)}} R^{(2), g}(\alpha) \\
& \triangleq-\varphi^{g}(\boldsymbol{r}, \boldsymbol{\Omega}) \frac{\partial^{2} \Sigma_{t}^{g}(\boldsymbol{t})}{\partial \alpha_{i_{2}} \partial \alpha_{i_{1}}}+\sum_{g^{\prime}=1}^{G} \int_{4 \pi} \mathrm{d} \boldsymbol{\Omega}^{\prime} \varphi^{g^{\prime}}\left(\boldsymbol{r}, \boldsymbol{\Omega}^{\prime}\right)\left\{\frac{\partial^{2} \Sigma_{s}^{g^{\prime} \rightarrow g}\left(\boldsymbol{s} ; \boldsymbol{\Omega}^{\prime} \rightarrow \boldsymbol{\Omega}\right)}{\partial \alpha_{i_{2}} \partial \alpha_{i_{1}}}\right. \\
& +\frac{\partial \chi^{g}}{\partial \alpha_{i_{2}}} \frac{\partial\left[\left(v \Sigma_{f}\right)^{g^{\prime}}\right]}{\partial \alpha_{i_{1}}}+\chi^{g} \frac{\partial^{2}\left[\left(v \Sigma_{f}\right)^{g^{\prime}}\right]}{\partial \alpha_{i_{2}} \partial \alpha_{i_{1}}}+\frac{\partial\left[\left(v \Sigma_{f}\right)^{g^{\prime}}\right]}{\partial \alpha_{i_{2}}} \frac{\partial \chi^{g}}{\partial \alpha_{i_{1}}} \\
& \left.+\left(v \Sigma_{f}\right)^{g^{\prime}} \frac{\partial^{2} \chi^{g}}{\partial \alpha_{i_{2}} \partial \alpha_{i_{1}}}\right\}-\psi_{2}^{(2), g}\left(i_{1} ; \boldsymbol{r}, \boldsymbol{\Omega}\right) \frac{\partial \Sigma_{t}^{g}(\boldsymbol{t})}{\partial \alpha_{i_{2}}}+\sum_{g^{\prime}=1}^{G} \int_{4 \pi} \mathrm{d} \boldsymbol{\Omega}^{\prime} \psi_{2}^{(2), g^{\prime}}\left(i_{1} ; \boldsymbol{r}, \boldsymbol{\Omega}^{\prime}\right) \\
& \times\left\{\frac{\partial \Sigma_{s}^{g^{\prime} \rightarrow g}\left(\boldsymbol{s} ; \boldsymbol{\Omega}^{\prime} \rightarrow \boldsymbol{\Omega}\right)}{\partial \alpha_{i_{2}}}+\frac{\partial\left[\left(v \Sigma_{f}\right)^{g^{\prime}}\right]}{\partial \alpha_{i_{2}}} \chi^{g}+\left(v \Sigma_{f}\right)^{g^{\prime}} \frac{\partial \chi^{g}}{\partial \alpha_{i_{2}}}\right\}, \\
& {\left[F_{32}^{(3), g}\right]^{*} \psi_{3}^{(3), g}\left(i_{1}, i_{2} ; \boldsymbol{r}, \boldsymbol{\Omega}\right)} \\
& \triangleq \psi_{3}^{(3), g}\left(i_{1}, i_{2} ; \boldsymbol{r}, \boldsymbol{\Omega}\right) \frac{\partial \Sigma_{t}^{g}(\boldsymbol{t})}{\partial \alpha_{i_{1}}}-\sum_{g^{\prime}=1}^{G} \int_{4 \pi} \mathrm{d} \boldsymbol{\Omega}^{\prime} \psi_{3}^{(3), g^{\prime}}\left(i_{1}, i_{2} ; \boldsymbol{r}, \boldsymbol{\Omega}\right) \\
& \times\left\{\frac{\partial \Sigma_{s}^{g^{\prime} \rightarrow g}\left(\boldsymbol{s} ; \boldsymbol{\Omega}^{\prime} \rightarrow \boldsymbol{\Omega}\right)}{\partial \alpha_{i_{1}}}+\frac{\partial\left[\left(v \Sigma_{f}\right)^{g^{\prime}}\right]}{\partial \alpha_{i_{1}}} \chi^{g}+\left(v \Sigma_{f}\right)^{g^{\prime}} \frac{\partial \chi^{g}}{\partial \alpha_{i_{1}}}\right\} \text {. }
\end{aligned}
$$

The particular forms taken on by the general expressions given Equations (25) through (33) when considering solely the group-averaged microscopic total cross sections are obtained specializing these expressions to the particular cases $\alpha_{i_{1}} \rightarrow t_{j}, \alpha_{i_{2}} \rightarrow t_{k}, \alpha_{i_{3}} \rightarrow t_{\ell}$. Thus, the expression in Equation (25) becomes:

$$
\begin{aligned}
& \left\{\frac{\partial Q^{(1), g}(\boldsymbol{\alpha} ; \varphi)}{\partial \alpha_{i_{2}}}\right\}_{\alpha_{i_{2}} \rightarrow t_{k}} \\
& \triangleq \frac{\partial Q^{g}(\boldsymbol{\alpha} ; r, \boldsymbol{\Omega})}{\partial t_{k}}-\frac{\partial}{\partial t_{k}}\left\{\boldsymbol{\Omega} \cdot \nabla \varphi^{g}(r, \boldsymbol{\Omega})+\Sigma_{t}^{g}(\boldsymbol{t}) \varphi^{g}(r, \boldsymbol{\Omega})\right. \\
& \left.-\sum_{g^{\prime}=1}^{G} \int_{4 \pi} \mathrm{d} \boldsymbol{\Omega}^{\prime} \varphi^{g^{\prime}}\left(r, \boldsymbol{\Omega}^{\prime}\right)\left[\Sigma_{s}^{g^{\prime} \rightarrow g}\left(\boldsymbol{s} ; \boldsymbol{\Omega}^{\prime} \rightarrow \boldsymbol{\Omega}\right)+\chi^{g}\left(v \Sigma_{f}\right)^{g^{\prime}}(\boldsymbol{f})\right]\right\} \\
& =-\varphi^{g}(r, \boldsymbol{\Omega}) \frac{\partial \Sigma_{t}^{g}(\boldsymbol{t})}{\partial \sigma_{t, i_{k}}^{g_{k}}}=-\varphi^{g}(r, \boldsymbol{\Omega}) \delta_{g_{k} g} N_{i_{k}, m_{k}} \cdot
\end{aligned}
$$


In view of the result obtained in Equation (34), it follows that the expression in Equation (26) vanishes, i.e.,

$$
\frac{\partial^{2} Q^{(1), g}(\alpha ; \varphi)}{\partial \alpha_{i_{3}} \partial \alpha_{i_{2}}}=0, \text { for } \alpha_{i_{2}} \rightarrow t_{k}, \alpha_{i_{3}} \rightarrow t_{\ell} .
$$

The expression in Equation (27) becomes:

$$
\begin{aligned}
& \left\{\frac{\partial Q^{(2), g}\left(\boldsymbol{\alpha} ; \psi^{(1), g}\right)}{\partial \alpha_{i_{2}}}\right\}_{\alpha_{i_{2} \rightarrow t_{k}}} \\
& \triangleq \frac{\partial \Sigma_{d}^{g}(\boldsymbol{\alpha} ; r, \boldsymbol{\Omega})}{\partial t_{k}}-\frac{\partial}{\partial t_{k}}\left\{-\boldsymbol{\Omega} \cdot \nabla \psi^{(1), g}(r, \boldsymbol{\Omega})+\Sigma_{t}^{g}(\boldsymbol{t}) \psi^{(1), g}(r, \boldsymbol{\Omega})\right. \\
& \left.\quad+\sum_{g^{\prime}=1}^{G} \int_{4 \pi} \mathrm{d} \boldsymbol{\Omega}^{\prime} \psi^{(1), g^{\prime}}\left(r, \boldsymbol{\Omega}^{\prime}\right)\left[\Sigma_{s}^{g \rightarrow g^{\prime}}\left(\boldsymbol{s} ; \boldsymbol{\Omega} \rightarrow \boldsymbol{\Omega}^{\prime}\right)+v \Sigma_{f}^{g}(\boldsymbol{f}) \chi^{g^{\prime}}\right]\right\} \\
& =-\psi^{(1), g}(r, \boldsymbol{\Omega}) \frac{\partial \Sigma_{t}^{g}(\boldsymbol{t})}{\partial \sigma_{t, i_{k}}^{g_{k}}}=-\psi^{(1), g}(r, \boldsymbol{\Omega}) \delta_{g_{k} g} N_{i_{k}, m_{k}} .
\end{aligned}
$$

In view of Equation (36), the expression in Equation (28) becomes:

$$
\left\{\nabla_{\psi_{2}^{(2)}\left(i_{1}\right)} R^{(2), g}(\boldsymbol{\alpha})\right\}_{\alpha_{i_{2} \rightarrow t_{k}}}=\frac{\partial Q^{(2), g}\left(\boldsymbol{\alpha} ; \psi^{(1), g}\right)}{\partial t_{k}}=-\psi^{(1), g}(r, \boldsymbol{\Omega}) \delta_{g_{k} g} N_{i_{k}, m_{k}} .
$$

The expression in Equation (31) becomes:

$$
\left\{\nabla_{\varphi} R^{(2), g}(\boldsymbol{\alpha})\right\}_{\alpha_{i_{2}} \rightarrow t_{k}} \triangleq-\psi_{1}^{(2), g}\left(i_{1} ; r, \boldsymbol{\Omega}\right) \frac{\partial \Sigma_{t}^{g}(\boldsymbol{t})}{\partial \sigma_{t, i_{k}}^{g_{k}}}=-\psi_{1}^{(2), g}\left(i_{1} ; r, \boldsymbol{\Omega}\right) \delta_{g_{k} g} N_{i_{k}, m_{k}} .
$$

The expression in Equation (29) becomes:

$$
\begin{aligned}
\{ & {\left.\left[F_{41}^{(3), g}\right]^{*} \psi_{4}^{(3), g}\left(i_{1}, i_{2} ; r, \boldsymbol{\Omega}\right)\right\}_{\alpha_{i_{1} \rightarrow t_{j}}} } \\
= & \psi_{4}^{(3), g}\left(i_{1}, i_{2} ; r, \boldsymbol{\Omega}\right) \frac{\partial \Sigma_{t}^{g}(\boldsymbol{t})}{\partial t_{j}}-\sum_{g^{\prime}=1}^{G} \int_{4 \pi} \mathrm{d} \boldsymbol{\Omega}^{\prime} \psi_{4}^{(3), g^{\prime}}\left(i_{1}, i_{2} ; r, \boldsymbol{\Omega}^{\prime}\right) \\
& \times\left\{\frac{\partial \Sigma_{s}^{g \rightarrow g^{\prime}}\left(\boldsymbol{s} ; \boldsymbol{\Omega} \rightarrow \boldsymbol{\Omega}^{\prime}\right)}{\partial t_{j}}+\frac{\partial\left[v \Sigma_{f}^{g}(\boldsymbol{f})\right]}{\partial t_{j}} \chi^{g^{\prime}}+v \Sigma_{f}^{g}(\boldsymbol{f}) \frac{\partial \chi^{g^{\prime}}}{\partial t_{j}}\right\} \\
= & \psi_{4}^{(3), g}\left(i_{1}, i_{2} ; r, \boldsymbol{\Omega}\right) \frac{\partial \Sigma_{t}^{g}(\boldsymbol{t})}{\partial \sigma_{t, i_{j}}^{g_{j}}}=\psi_{4}^{(3), g}\left(i_{1}, i_{2} ; r, \boldsymbol{\Omega}\right) \delta_{g_{j} g} N_{i_{j}, m_{j}} .
\end{aligned}
$$

In view of Equation (34), the expression in Equation (30) becomes:

$$
\begin{aligned}
\left\{\nabla_{\psi_{1}^{(2)}\left(i_{1}\right)} R^{(2), g}(\boldsymbol{\alpha})\right\}_{\alpha_{i_{2} \rightarrow t_{k}}} & =\frac{\partial Q^{(1), g}(\boldsymbol{\alpha} ; \varphi)}{\partial t_{k}} \\
& =-\varphi^{g}(r, \boldsymbol{\Omega}) \frac{\partial \Sigma_{t}^{g}(\boldsymbol{t})}{\partial \sigma_{t, i_{k}}^{g_{k}}} \\
& =-\varphi^{g}(r, \boldsymbol{\Omega}) \delta_{g_{k} g} N_{i_{k}, m_{k}} .
\end{aligned}
$$

The expression in Equation (32) becomes: 


$$
\begin{aligned}
& \left\{\nabla_{\psi^{(1)}} R^{(2), g}(\boldsymbol{\alpha})\right\}_{\substack{\alpha_{i_{i} \rightarrow t_{j}} \\
\alpha_{i_{2}} \rightarrow t_{k}}} \\
& =-\varphi^{g}(r, \boldsymbol{\Omega}) \frac{\partial^{2} \Sigma_{t}^{g}(\boldsymbol{t})}{\partial t_{k} \partial t_{j}}+\sum_{g^{\prime}=1}^{G} \int_{4 \pi} \mathrm{d} \boldsymbol{\Omega}^{\prime} \varphi^{g^{\prime}}\left(r, \boldsymbol{\Omega}^{\prime}\right) \\
& \times\left\{\frac{\partial^{2} \Sigma_{s}^{g^{\prime} \rightarrow g}\left(s ; \boldsymbol{\Omega}^{\prime} \rightarrow \boldsymbol{\Omega}\right)}{\partial t_{k} \partial t_{j}}+\frac{\partial \chi^{g}}{\partial t_{k}} \frac{\partial\left[\left(v \Sigma_{f}\right)^{g^{\prime}}\right]}{\partial t_{j}}+\chi^{g} \frac{\partial^{2}\left[\left(v \Sigma_{f}\right)^{g^{\prime}}\right]}{\partial t_{k} \partial t_{j}}\right. \\
& \left.+\frac{\partial\left[\left(v \Sigma_{f}\right)^{g^{\prime}}\right]}{\partial t_{k}} \frac{\partial \chi^{g}}{\partial t_{j}}+\left(v \Sigma_{f}\right)^{g^{\prime}} \frac{\partial^{2} \chi^{g}}{\partial t_{k} \partial t_{j}}\right\} \\
& -\psi_{2}^{(2), g}\left(i_{1} ; r, \boldsymbol{\Omega}\right) \frac{\partial \Sigma_{t}^{g}(\boldsymbol{t})}{\partial t_{k}}+\sum_{g^{\prime}=1}^{G} \int_{4 \pi} \mathrm{d} \boldsymbol{\Omega}^{\prime} \psi_{2}^{(2), g^{\prime}}\left(i_{1} ; r, \boldsymbol{\boldsymbol { S } ^ { \prime }}\right) \\
& \times\left\{\frac{\partial \Sigma_{s}^{g^{\prime} \rightarrow g}\left(\boldsymbol{s} ; \boldsymbol{\Omega}^{\prime} \rightarrow \boldsymbol{\Omega}\right)}{\partial t_{k}}+\frac{\partial\left[\left(v \Sigma_{f}\right)^{g^{\prime}}\right]}{\partial t_{k}} \chi^{g}+\left(v \Sigma_{f}\right)^{g^{\prime}} \frac{\partial \chi^{g}}{\partial t_{k}}\right\} \\
& =-\psi_{2}^{(2), g}\left(i_{1} ; r, \boldsymbol{\Omega}\right) \frac{\partial \Sigma_{t}^{g}(\boldsymbol{t})}{\partial t_{k}}=-\psi_{2}^{(2), g}\left(i_{1} ; r, \boldsymbol{\Omega}\right) \frac{\partial \Sigma_{t}^{g}(\boldsymbol{t})}{\partial \sigma_{t, i_{k}}^{g_{k}}} \\
& =-\psi_{2}^{(2), g}\left(i_{1} ; r, \boldsymbol{\Omega}\right) \delta_{g_{k} g} N_{i_{k}, m_{k}} \cdot
\end{aligned}
$$

The expression in Equation (33) becomes:

$$
\begin{aligned}
\{ & {\left.\left[F_{32}^{(3), g}\right]^{*} \psi_{3}^{(3), g}\left(i_{1}, i_{2} ; r, \boldsymbol{\Omega}\right)\right\}_{\substack{\alpha_{i_{i} \rightarrow t_{j}} \\
\alpha_{i_{2} \rightarrow t_{k}}}} } \\
= & \psi_{3}^{(3), g}(j, k ; r, \boldsymbol{\Omega}) \frac{\partial \Sigma_{t}^{g}(\boldsymbol{t})}{\partial t_{j}}-\sum_{g^{\prime}=1}^{G} \int \mathrm{d} \boldsymbol{\Omega}^{\prime} \psi_{3}^{(3), g^{\prime}}(j, k ; r, \boldsymbol{\Omega}) \\
& \times\left\{\frac{\partial \Sigma_{s}^{g^{\prime} \rightarrow g}\left(s ; \boldsymbol{\Omega}^{\prime} \rightarrow \boldsymbol{\Omega}\right)}{\partial t_{j}}+\frac{\partial\left[\left(v \Sigma_{f}\right)^{g^{\prime}}\right]}{\partial t_{j}} \chi^{g}+\left(v \Sigma_{f}\right)^{g^{\prime}} \frac{\partial \chi^{g}}{\partial t_{j}}\right\} \\
= & \psi_{3}^{(3), g}(j, k ; r, \boldsymbol{\Omega}) \frac{\partial \Sigma_{t}^{g}(\boldsymbol{t})}{\partial \sigma_{t, i_{j}}^{g_{j}}}=\psi_{3}^{(3), g}(j, k ; r, \boldsymbol{\Omega}) \delta_{g_{j} g} N_{i_{j}, m_{j}} .
\end{aligned}
$$

Collecting the results obtained in Equations (34) through (42), inserting them into Equations (16) through (23) and recalling that $i_{1} \rightarrow j, i_{2} \rightarrow k$ yields the following particular form for the $3^{\text {rd }}$-LASS, specialized for computing the $3^{\text {rd }}$-level adjoint functions needed for computing the $3^{\text {rd }}$-order sensitivities of the PERP leakage response to the group-averaged total microscopic cross sections:

$$
\begin{aligned}
A^{g}(\boldsymbol{\alpha}) \psi_{4}^{(3), g}(j, k ; \boldsymbol{r}, \boldsymbol{\Omega})=-\psi^{(1), g}(r, \boldsymbol{\Omega}) \delta_{g_{k} g} N_{i_{k}, m_{k}}, j=1, \cdots, J_{\sigma t}, k=1, \cdots, j, \\
\psi_{4}^{(3), g}\left(j, k ; r_{d}, \boldsymbol{\Omega}\right)=0, \boldsymbol{\Omega} \cdot \boldsymbol{n}>0, j=1, \cdots, J_{\sigma t}, k=1, \cdots, j, \\
A^{g}(\boldsymbol{\alpha}) \psi_{1}^{(3), g}(j, k ; r, \boldsymbol{\Omega}) \\
=-\left[\psi_{1}^{(2), g}(j ; r, \boldsymbol{\Omega}) \delta_{g_{k} g} N_{i_{k}, m_{k}}+\psi_{4}^{(3), g}(j, k ; r, \boldsymbol{\Omega}) \delta_{g_{j} g} N_{i_{j}, m_{j}}\right],
\end{aligned}
$$




$$
\begin{gathered}
\psi_{1}^{(3), g}\left(j, k ; r_{d}, \boldsymbol{\Omega}, E\right)=0, \boldsymbol{\Omega} \cdot \boldsymbol{n}>0, j=1, \cdots, J_{\sigma t}, k=1, \cdots, j, \\
B^{g}(\boldsymbol{\alpha}) \psi_{3}^{(3), g}(j, k ; r, \boldsymbol{\Omega})=-\varphi^{g}(r, \boldsymbol{\Omega}) \delta_{g_{k} g} N_{i_{k}, m_{k}}, j=1, \cdots, J_{\sigma t}, k=1, \cdots, j, \\
\psi_{3}^{(3), g}\left(j, k ; r_{d}, \boldsymbol{\Omega}\right)=0, \boldsymbol{\Omega} \cdot \boldsymbol{n}<0, j=1, \cdots, J_{\sigma t}, k=1, \cdots, j, \\
B^{g}(\alpha) \psi_{2}^{(3), g}(j, k ; r, \boldsymbol{\Omega}) \\
=-\left[\psi_{2}^{(2), g}(j ; r, \boldsymbol{\Omega}) \delta_{g_{k} g} N_{i_{k}, m_{k}}+\psi_{3}^{(3), g}(j, k ; r, \boldsymbol{\Omega}) \delta_{g_{j} g} N_{i_{j}, m_{j}}\right], \\
\psi_{2}^{(3), g}\left(j, k ; r_{d}, \boldsymbol{\Omega}\right)=0, \boldsymbol{\Omega} \cdot \boldsymbol{n}<0, j=1 \cdots, J_{\sigma t}, k=1, \cdots, j .
\end{gathered}
$$

The $2^{\text {nd }}$-level adjoint functions $\psi_{1}^{(2), g}(j ; r, \boldsymbol{\Omega})$ and $\psi_{2}^{(2), g}(j ; r, \boldsymbol{\Omega})$ are the solutions of the following $2^{\text {nd }}$-LASS [5]:

$$
\begin{gathered}
A^{g}(\boldsymbol{\alpha}) \psi_{1}^{(2), g}(j ; r, \boldsymbol{\Omega})=-\delta_{g_{j} g} N_{i_{j}, m_{j}} \psi^{(1), g}(r, \boldsymbol{\Omega}), j=1, \cdots, J_{\sigma t} ; g=1, \cdots, G, \\
\psi_{1, j}^{(2), g}\left(r_{d}, \boldsymbol{\Omega}\right)=0, \boldsymbol{\Omega} \cdot \boldsymbol{n}>0 ; j=1, \cdots, J_{\sigma t} ; g=1, \cdots, G, \\
B^{g}(\boldsymbol{\alpha}) \psi_{2}^{(2), g}(j ; r, \boldsymbol{\Omega})=-\delta_{g_{j} g} N_{i_{j}, m_{j}} \varphi^{g}(r, \boldsymbol{\Omega}), j=1, \cdots, J_{\sigma t} ; g=1, \cdots, G, \\
\psi_{2, j}^{(2), g}\left(r_{d}, \boldsymbol{\Omega}\right)=0, \boldsymbol{\Omega} \cdot \boldsymbol{n}<0 ; j=1, \cdots, J_{\sigma t} ; g=1, \cdots, G,
\end{gathered}
$$

while the $1^{\text {st }}$-level adjoint function $\psi^{(1), g}(r, \Omega)$ is the solution of the following

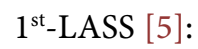

$$
\begin{gathered}
A^{g}(\boldsymbol{\alpha}) \psi^{(1), g}(r, \boldsymbol{\Omega})=\boldsymbol{\Omega} \cdot \boldsymbol{n} \delta\left(r-r_{d}\right), g=1, \cdots, G, \\
\psi^{(1), g}\left(r_{d}, \boldsymbol{\Omega}\right)=0, \boldsymbol{\Omega} \cdot \boldsymbol{n}>0, g=1, \cdots, G .
\end{gathered}
$$

Next, each of the quantities on the right-side of Equation (14) will be specialized to obtain the specific expression for computing the $3^{\text {rd }}$-order sensitivities of the leakage response in the PERP benchmark to the 180 total cross sections $\sigma_{t, i}^{g}, i=1, \cdots, I=6 ; g=1, \cdots, G=30$ defined in Equation (8). Thus, in view of Equation (10), it follows that the first term on the right-side of Equation (14) vanishes, since

$$
\frac{\partial^{3} \Sigma_{d}^{g}(\boldsymbol{\alpha})}{\partial \alpha_{i_{3}} \partial \alpha_{i_{2}} \partial \alpha_{i_{1}}}=\frac{\partial^{3} \Sigma_{d}^{g}(\boldsymbol{\alpha})}{\partial t_{j} \partial t_{k} \partial t_{\ell}}=0
$$

In view of Equation (35), the second term on the right-side of Equation (14) vanishes, i.e.,

$$
\left\langle\psi^{(1), g}(r, \boldsymbol{\Omega}), \frac{\partial^{3} Q^{(1), g}(\boldsymbol{\alpha})}{\partial \alpha_{i_{3}} \partial \alpha_{i_{2}} \partial \alpha_{i_{1}}}\right\rangle_{(1)}=0 .
$$

In view of Equation (35), the third term on the right-side of Equation (14) vanishes, i.e.,

$$
\left\langle\psi_{1}^{(2), g}\left(i_{1} ; r, \boldsymbol{\Omega}\right), \frac{\partial^{2} Q^{(1), g}(\boldsymbol{\alpha})}{\partial \alpha_{i_{3}} \partial \alpha_{i_{2}}}\right\rangle_{(1)}=0 .
$$

In view of Equation (36), the fourth term on the right-side of Equation (14) vanishes, i.e., 


$$
\left\langle\psi_{2}^{(2), g}\left(i_{1} ; r, \boldsymbol{\Omega}\right), \frac{\partial^{2} Q^{(2), g}(\boldsymbol{\alpha})}{\partial \alpha_{i_{3}} \partial \alpha_{i_{2}}}\right\rangle_{(1)}=0 .
$$

In view of Equation (34), the fifth term on the right-side of Equation (14) takes on the following particular form:

$$
\begin{aligned}
& \left\langle\psi_{1}^{(3), g}\left(i_{1}, i_{2} ; r, \boldsymbol{\Omega}\right), \frac{\partial Q^{(1), g}(\boldsymbol{\alpha})}{\partial \alpha_{i_{3}}}\right\rangle_{(1)} \\
& =-\left\langle\psi_{1}^{(3), g}\left(i_{1}, i_{2} ; r, \boldsymbol{\Omega}\right), \varphi^{g}(r, \boldsymbol{\Omega}) \delta_{g_{\ell} g} N_{i_{\ell}, m_{\ell}}\right\rangle_{(1)} .
\end{aligned}
$$

In view of Equation (36), the sixth term on the right-side of Equation (14) takes on the following particular form:

$$
\begin{aligned}
& \left\langle\psi_{2}^{(3), g}\left(i_{1}, i_{2} ; r, \boldsymbol{\Omega}\right), \frac{\partial Q^{(2), g}(\boldsymbol{\alpha})}{\partial \alpha_{i_{3}}}\right\rangle_{(1)} \\
& =-\left\langle\psi_{2}^{(3), g}\left(i_{1}, i_{2} ; r, \boldsymbol{\Omega}\right), \psi^{(1), g}(r, \boldsymbol{\Omega}) \delta_{g_{\ell} g} N_{i_{\ell}, m_{\ell}}\right\rangle_{(1)},
\end{aligned}
$$

The seventh term on the right-side of Equation (14) takes on the following particular form:

$$
\begin{aligned}
& \left\langle\psi_{3}^{(3), g}\left(i_{1}, i_{2} ; r, \boldsymbol{\Omega}\right), \frac{\partial Q_{1}^{(3), g}(\boldsymbol{\alpha})}{\partial \alpha_{i_{3}}}\right\rangle_{(1)} \\
& =-\left\langle\psi_{3}^{(3), g}\left(i_{1}, i_{2} ; r, \boldsymbol{\Omega}\right), \psi_{1}^{(2), g}\left(i_{1} ; r, \boldsymbol{\Omega}\right) \delta_{g_{\ell} g} N_{i_{\ell}, m_{\ell}}\right\rangle_{(1)},
\end{aligned}
$$

since

$$
\begin{aligned}
& \frac{\partial Q_{1}^{(3), g}\left[\boldsymbol{\alpha} ; r, \boldsymbol{\Omega} ; \psi_{1}^{(2), g}\left(i_{1}\right)\right]}{\partial \alpha_{i_{3}}} \\
& \triangleq-\frac{\partial\left[A^{g}(\boldsymbol{\alpha}) \psi_{1}^{(2), g}\left(i_{1} ; r, \boldsymbol{\Omega}\right)\right]}{\partial \alpha_{i_{3}}}+\frac{\partial\left[\nabla_{\varphi} R^{(1)}\left(\boldsymbol{\alpha} ; \psi^{(1), g}\right)\right]}{\partial \alpha_{i_{3}}} \\
& =-\psi_{1}^{(2), g}\left(i_{1} ; r, \boldsymbol{\Omega}\right) \frac{\partial \Sigma_{t}^{g}(\boldsymbol{t})}{\partial \sigma_{t, i_{l}}^{g_{l}}}=-\psi_{1}^{(2), g}\left(i_{1} ; r, \boldsymbol{\Omega}\right) \delta_{g_{\ell} g} N_{i_{\ell}, m_{\ell}} .
\end{aligned}
$$

The result obtained in Equation (64) stems from the fact that repeating the derivation shown in Equation (36) yields the results $\partial\left[A(\boldsymbol{\alpha}) \psi_{1}^{(2), g}\left(i_{1} ; r, \boldsymbol{\Omega}\right)\right] / \partial \alpha_{i_{3}}=\psi_{1}^{(2), g}\left(i_{1} ; r, \boldsymbol{\Omega}\right)\left[\partial \Sigma_{t}^{g}(\boldsymbol{t}) / \partial \sigma_{t, i_{\ell}}^{g_{l_{l}}}\right]$, and from the fact that the second term on the right side of Equation (64) vanishes, as shown below:

$$
\begin{aligned}
& \left\{\frac{\partial\left[\nabla_{\varphi} R^{(1)}\left(\boldsymbol{\alpha} ; \psi^{(1), g}\right)\right]}{\partial \alpha_{i_{3}}}\right\} \underbrace{}_{\substack{\alpha_{i_{1} \rightarrow t_{j}} \\
\alpha_{i_{3} \rightarrow t_{\ell}}}}{ }^{\triangleq} \frac{\partial^{2} \Sigma_{d}^{g}(\boldsymbol{\alpha} ; r)}{\partial t_{\ell} \partial t_{j}}-\psi^{(1), g}(r, \boldsymbol{\Omega}) \frac{\partial^{2} \Sigma_{t}^{g}(\boldsymbol{t})}{\partial t_{\ell} \partial t_{j}}+\sum_{g^{\prime}=1}^{G} \int_{4 \pi} \mathrm{d} \boldsymbol{\Omega}^{\prime} \psi^{(1), g^{\prime}}\left(r, \boldsymbol{\Omega}^{\prime}\right)
\end{aligned}
$$




$$
\begin{aligned}
& \times\left\{\frac{\partial^{2} \Sigma_{s}^{g \rightarrow g^{\prime}}\left(s ; \boldsymbol{\Omega}^{\prime} \rightarrow \boldsymbol{\Omega}\right)}{\partial t_{\ell} \partial t_{j}}+\chi^{g^{\prime}} \frac{\partial^{2}\left[\left(v \Sigma_{f}\right)^{g}\right]}{\partial t_{\ell} \partial t_{j}}\right. \\
& \left.+\frac{\partial\left[\left(v \Sigma_{f}\right)^{g}\right]}{\partial t_{j}} \frac{\partial \chi^{g^{\prime}}}{\partial t_{\ell}}+\frac{\partial\left[\left(v \Sigma_{f}\right)^{g}\right]}{\partial t_{\ell}} \frac{\partial \chi^{g^{\prime}}}{\partial t_{j}}+\left(v \Sigma_{f}\right)^{g} \frac{\partial^{2} \chi^{g^{\prime}}}{\partial t_{\ell} \partial t_{j}}\right\}=0 .
\end{aligned}
$$

The eighth term on the right-side of Equation (14) takes on the following particular form:

$$
\begin{aligned}
& \left\langle\psi_{4}^{(3), g}\left(i_{1}, i_{2} ; r, \boldsymbol{\Omega}\right), \frac{\partial Q_{2}^{(3), g}(\boldsymbol{\alpha})}{\partial \alpha_{i_{3}}}\right\rangle_{(1)} \\
& =-\left\langle\psi_{4}^{(3), g}\left(i_{1}, i_{2} ; r, \boldsymbol{\Omega}\right), \psi_{2}^{(2), g}\left(i_{1} ; r, \boldsymbol{\Omega}\right) \delta_{g_{\ell} g} N_{i_{\ell}, m_{\ell}}\right\rangle_{(1)}
\end{aligned}
$$

since

$$
\begin{aligned}
& \frac{\partial Q_{2}^{(3), g}\left[\boldsymbol{\alpha} ; r, \boldsymbol{\Omega} ; \psi_{2}^{(2), g}\left(i_{1}\right)\right]}{\partial \alpha_{i_{3}}} \\
& \triangleq-\frac{\partial\left[B^{g}(\boldsymbol{\alpha}) \psi_{2}^{(2), g}\left(i_{1} ; r, \boldsymbol{\Omega}\right)\right]}{\partial \alpha_{i_{3}}}+\frac{\partial\left[\nabla_{\psi^{(1)}} R^{(1)}(\boldsymbol{\alpha} ; \varphi)\right]}{\partial \alpha_{i_{3}}} \\
& =-\psi_{2}^{(2), g}\left(i_{1} ; r, \boldsymbol{\Omega}\right) \frac{\partial \Sigma_{t}^{g}(\boldsymbol{t})}{\partial \sigma_{t, i_{\ell}}^{g_{l_{\ell}}}} \\
& =-\psi_{2}^{(2), g}\left(i_{1} ; r, \boldsymbol{\Omega}\right) \delta_{g_{\ell g} N_{i_{\ell}, m_{\ell}},}
\end{aligned}
$$

as can be shown by repeating the derivations used in Equation (34) to obtain the result $\partial\left[B^{g}(\boldsymbol{\alpha}) \psi_{2}^{(2), g}\left(i_{1} ; r, \boldsymbol{\Omega}\right)\right] / \partial \alpha_{i_{3}}=\psi_{2}^{(2), g}\left(i_{1} ; r, \boldsymbol{\Omega}\right)\left[\partial \Sigma_{t}^{g}(\boldsymbol{\alpha} ; r) / \partial \sigma_{t, i_{\ell}}^{g_{l}}\right]$, and by noting that

$$
\begin{aligned}
& \left\{\frac{\partial\left[\nabla_{\psi^{(1)}} R^{(1)}(\boldsymbol{\alpha} ; \varphi)\right]}{\partial \alpha_{i_{3}}}\right\} \\
& =\frac{\partial^{2} Q^{g}(\boldsymbol{\alpha} ; r, \boldsymbol{\Omega})}{\partial t_{\ell} \partial t_{j}}-\varphi^{g}(r, \boldsymbol{\Omega}) \frac{\partial^{2} \Sigma_{t}^{g}(\boldsymbol{t})}{\partial t_{\ell} \partial t_{j}} \\
& +\sum_{g^{\prime}=1}^{G} \int_{4 \pi} \mathrm{d} \boldsymbol{\Omega}^{\prime} \varphi^{g^{\prime}}\left(r, \boldsymbol{\Omega}^{\prime}\right) \\
& \times\left\{\frac{\partial^{2} \Sigma_{s}^{g^{\prime} \rightarrow g}\left(\boldsymbol{s} ; \boldsymbol{\Omega}^{\prime} \rightarrow \boldsymbol{\Omega}\right)}{\partial t_{\ell} \partial t_{j}}+\frac{\partial \chi^{g}}{\partial t_{\ell}} \frac{\partial\left[\left(v \Sigma_{f}\right)^{g^{\prime}}\right]}{\partial t_{j}}\right. \\
& \left.+\chi^{g} \frac{\partial^{2}\left[\left(v \Sigma_{f}\right)^{g^{\prime}}\right]}{\partial t_{\ell} \partial t_{j}}+\frac{\partial\left[\left(v \Sigma_{f}\right)^{g^{\prime}}\right]}{\partial t_{\ell}} \frac{\partial \chi^{g}}{\partial t_{j}}+\left(v \Sigma_{f}\right)^{g^{\prime}} \frac{\partial^{2} \chi^{g}}{\partial t_{\ell} \partial t_{j}}\right\}=0 .
\end{aligned}
$$

Collecting the results obtained in Equations (57)-(63), and (66), and replacing them in Equation (14) yields the following expression: 


$$
\begin{aligned}
& \frac{\partial^{3} L(\boldsymbol{\alpha})}{\partial t_{j} \partial t_{k} \partial t_{\ell}}=-\left\{\left\langle\psi_{1}^{(3), g}(j, k ; r, \boldsymbol{\Omega}), \varphi^{g}(r, \boldsymbol{\Omega})\right\rangle_{(1)}\right. \\
&+\left\langle\psi_{2}^{(3), g}(j, k ; r, \boldsymbol{\Omega}), \psi^{(1), g}(r, \boldsymbol{\Omega})\right\rangle_{(1)} \\
&+\left\langle\psi_{3}^{(3), g}(j, k ; r, \boldsymbol{\Omega}), \psi_{1}^{(2), g}(j ; r, \boldsymbol{\Omega})\right\rangle_{(1)} \\
&\left.+\left\langle\psi_{4}^{(3), g}(j, k ; r, \boldsymbol{\Omega}), \psi_{2}^{(2), g}(j ; r, \boldsymbol{\Omega})\right\rangle_{(1)}\right\} \delta_{g_{\ell} g} N_{i_{\ell}, m_{\ell}}, \\
& \text { for } j=1, \cdots, J_{\sigma t}, k=1, \cdots, j, \ell=1, \cdots, k .
\end{aligned}
$$

\subsection{Computing $\partial^{3} L(\alpha) / \partial t_{j} \partial t_{k} \partial t_{\ell}, j, k, \ell=1, \cdots, J_{\sigma t}$ by Using the}

Second-Order Sensitivities $\partial^{2} L(\alpha) / \partial t_{j} \partial t_{k}, j, k=1, \cdots, J_{\sigma t}$ of the PERP Leakage Response to Total Cross Sections

In Ref. [5], the specific $2^{\text {nd }}$-order sensitivities of the PERP leakage response to the group-averaged microscopic total cross sections were shown to have the following expression:

$$
\begin{aligned}
& \frac{\partial^{2} L}{\partial t_{j} \partial t_{k}}=\frac{\partial^{2} L}{\partial \sigma_{t, i_{j}}^{g_{j}} \partial \sigma_{t, i_{k}}^{g_{k}}}=-N_{i_{k}, m_{k}} \int_{V} \mathrm{~d} V \int_{4 \pi} \mathrm{d} \boldsymbol{\Omega}\left[\psi_{2, j}^{(2), g_{k}}(r, \boldsymbol{\Omega}) \psi^{(1), g_{k}}(r, \boldsymbol{\Omega})\right. \\
& \left.+\psi_{1, j}^{(2), g_{k}}(r, \boldsymbol{\Omega}) \varphi^{g_{k}}(r, \boldsymbol{\Omega})\right], \text { for } j=1, \cdots, J_{\sigma t} ; k=1, \cdots, J_{\sigma t}, g=1, \cdots, G,
\end{aligned}
$$

where the first-level adjoint functions $\psi^{(1), g}(r, \Omega), g=1, \cdots, G$ are the solutions of the $1^{\text {st }}$-Level Adjoint Sensitivity System ( $1^{\text {st }}$ LASS) show in Equations (55) and (56), while the $2^{\text {nd }-l e v e l ~ a d j o i n t ~ f u n c t i o n s ~} \psi_{1, j}^{(2), g}(r, \boldsymbol{\Omega})$ and $\psi_{2, j}^{(2), g}(r, \boldsymbol{\Omega})$ are the solutions of the $2^{\text {nd }}$-LASS presented in Equations (51) through (54).

The total G-differential of Equation (70) provides the $3^{\text {rd }}$-order sensitivities of the leakage response involving the $2^{\text {nd }}$-order derivatives to the total cross sections. Since this work is limited to computing the sensitivities of the PERP leakage response solely with respect to the group-averaged microscopic total cross sections, it follows that the sensitivities $\partial^{3} L(\alpha) / \partial t_{j} \partial t_{k} \partial t_{\ell}, j, k, \ell=1, \cdots, J_{\sigma t}$ are obtained by taking the following G-differential of Equation (70), limited to variations just in the group-averaged total microscopic cross sections, namely:

$$
\begin{aligned}
\delta\left(\frac{\partial^{2} L}{\partial t_{j} \partial t_{k}}\right)= & -N_{i_{k}, m_{k}} \int_{V} \mathrm{~d} V \int_{4 \pi} \mathrm{d} \boldsymbol{\Omega}\left[\delta \psi_{2, j}^{(2), g_{k}}(r, \boldsymbol{\Omega}) \psi^{(1), g_{k}}(r, \boldsymbol{\Omega})\right. \\
& +\psi_{2, j}^{(2), g_{k}}(r, \boldsymbol{\Omega}) \delta \psi^{(1), g_{k}}(r, \boldsymbol{\Omega})+\delta \psi_{1, j}^{(2), g_{k}}(r, \boldsymbol{\Omega}) \varphi^{g_{k}}(r, \boldsymbol{\Omega}) \\
& \left.+\psi_{1, j}^{(2), g_{k}}(r, \boldsymbol{\Omega}) \delta \varphi^{g_{k}}(r, \boldsymbol{\Omega})\right] \\
= & \sum_{\ell=1}^{J_{\sigma t}} \frac{\partial^{3} L(\boldsymbol{\alpha})}{\partial t_{j} \partial t_{k} \partial t_{\ell}} \delta t_{\ell}, \text { for } j, k=1, \cdots, J_{\sigma t},
\end{aligned}
$$

where the functions $\delta \psi^{(1), g_{k}}(r, \boldsymbol{\Omega})$ and $\delta \varphi^{g_{k}}(r, \boldsymbol{\Omega})$ are the solutions of the $1^{\text {st }}$ LFSS and, respectively, $2^{\text {nd }}-$ LFSS, which are reproduced below from Ref. [2], omitting the subscript " $k$ ":

$$
B^{g}(\boldsymbol{\alpha}) \delta \varphi^{g}(\boldsymbol{r}, \boldsymbol{\Omega})=Q^{(1), g}(\boldsymbol{\alpha}, \varphi ; \delta \boldsymbol{\alpha}), g=1, \cdots, G,
$$




$$
\begin{gathered}
\delta \varphi^{g}\left(r_{d}, \boldsymbol{\Omega}\right)=0, \boldsymbol{\Omega} \cdot \boldsymbol{n}<0, g=1, \cdots, G, \\
A^{g}(\boldsymbol{\alpha}) \delta \psi^{(1), g}(r, \boldsymbol{\Omega})=Q^{(2), g}\left(\boldsymbol{\alpha}, \psi^{(1)} ; \delta \boldsymbol{\alpha}\right), g=1, \cdots, G, \\
\delta \psi^{(1), g}\left(r_{d}, \boldsymbol{\Omega}\right)=0, \boldsymbol{\Omega} \cdot \boldsymbol{n}>0,
\end{gathered}
$$

while the functions $\delta \psi_{1, j}^{(2), g_{k}}$ and $\delta \psi_{2, j}^{(2), g_{k}}$ are the solutions of the G-differentiated $2^{\text {nd }}$-LASS defined by Equations (51) through (54), namely:

$$
\begin{gathered}
A^{g}(\boldsymbol{\alpha})\left[\delta \psi_{1, j}^{(2), g}(r, \boldsymbol{\Omega})\right]+\delta_{g_{j} g} N_{i_{j}, m_{j}}\left[\delta \psi^{(1), g}(r, \boldsymbol{\Omega})\right]=Q_{1}^{(3), g}\left[\boldsymbol{\alpha}, \psi_{1}^{(2)}\left(i_{1}\right) ; \delta \boldsymbol{\alpha}\right], \\
\delta \psi_{1, j}^{(2), g}\left(r_{d}, \boldsymbol{\Omega}\right)=0, \boldsymbol{\Omega} \cdot \boldsymbol{n}>0, j=1, \cdots, J_{\sigma t}, g=1, \cdots, G \\
B^{g}(\boldsymbol{\alpha})\left[\delta \psi_{2, j}^{(2), g}(r, \boldsymbol{\Omega})\right]+\delta_{g_{j} g} N_{i_{j}, m_{j}}\left[\delta \varphi^{g}(r, \boldsymbol{\Omega})\right]=Q_{2}^{(3)}\left[\boldsymbol{\alpha}, \psi_{2}^{(2)}\left(i_{1}\right) ; \delta \boldsymbol{\alpha}\right], \\
\delta \psi_{2, j}^{(2), g}\left(r_{d}, \boldsymbol{\Omega}\right)=0, \boldsymbol{\Omega} \cdot \boldsymbol{n}<0, j=1, \cdots, J_{\sigma t}, \quad g=1, \cdots, G .
\end{gathered}
$$

The source $Q^{(1), g}(\boldsymbol{\alpha}, \varphi ; \delta \boldsymbol{\alpha})$ on the right side in Equation (72) is defined as follows:

$$
Q^{(1), g}(\alpha, \varphi ; \delta \boldsymbol{\alpha}) \triangleq \sum_{i_{3}=1}^{J_{\sigma t}} \frac{\partial Q^{(1), g}(\boldsymbol{\alpha}, \varphi)}{\partial \alpha_{i_{3}}} \delta \alpha_{i_{3}},
$$

where, in view of Equation (34),

$$
\left\{\frac{\partial Q^{(1), g}(\boldsymbol{\alpha}, \varphi)}{\partial \alpha_{i_{3}}}\right\}_{\alpha_{i_{3}}=t_{l}} \triangleq-\varphi^{g}(r, \boldsymbol{\Omega}) \frac{\partial \Sigma_{t}^{g}(\boldsymbol{t})}{\partial \sigma_{t, i_{l}}^{g_{l}}}=-\varphi^{g}(r, \boldsymbol{\Omega}) \delta_{g_{l} g} N_{i_{l}, m_{l}} .
$$

The source $Q^{(2), g}\left(\boldsymbol{\alpha}, \psi^{(1)} ; \delta \boldsymbol{\alpha}\right)$ on the right side in Equation (74) is defined as follows:

$$
Q^{(2), g}\left(\alpha, \psi^{(1)} ; \delta \boldsymbol{\alpha}\right) \triangleq \sum_{i_{3}=1}^{J_{\sigma t}} \frac{\partial Q^{(2), g}\left(\alpha, \psi^{(1)}\right)}{\partial \alpha_{i_{3}}} \delta \alpha_{i_{3}},
$$

where, in view of Equation (36),

$$
\left\{\frac{\partial \boldsymbol{Q}^{(2), g}\left(\boldsymbol{\alpha}, \psi^{(1)}\right)}{\partial \alpha_{i_{3}}}\right\}_{\alpha_{i_{3}=t_{l}}} \triangleq-\psi^{(1), g}(r, \boldsymbol{\Omega}) \frac{\partial \Sigma_{t}^{g}(\boldsymbol{t})}{\partial \sigma_{t, i_{l}}^{g_{l}}}=-\psi^{(1), g}(r, \boldsymbol{\Omega}) \delta_{g_{l} g} N_{i_{l}, m_{l}} .
$$

The source $Q_{1}^{(3), g}\left[\boldsymbol{\alpha}, \psi_{1}^{(2)}\left(i_{1}\right) ; \delta \boldsymbol{\alpha}\right]$ on the right side in Equation (76) is defined as follows:

$$
Q_{1}^{(3), g}\left[\boldsymbol{\alpha}, \psi_{1}^{(2)}\left(i_{1}\right) ; \delta \boldsymbol{\alpha}\right] \triangleq \sum_{i_{3}=1}^{J_{\sigma t}} \frac{\partial Q_{1}^{(3), g}\left[\boldsymbol{\alpha}, \psi_{1}^{(2)}\left(i_{1}\right)\right]}{\partial \alpha_{i_{3}}} \delta \alpha_{i_{3}},
$$

where, in view of Equation (64),

$$
\begin{aligned}
& \left\{\frac{\partial Q_{1}^{(3), g}\left[\boldsymbol{\alpha}, \psi_{1}^{(2)}\left(i_{1}\right)\right]}{\partial \alpha_{i_{3}}}\right\}_{\alpha_{i_{3}=t_{l}}} \\
& \triangleq-\frac{\partial\left[A^{g}(\boldsymbol{\alpha}) \psi_{1}^{(2), g}\left(i_{1} ; \boldsymbol{r}, \boldsymbol{\Omega}\right)\right]}{\partial t_{l}}-\frac{\partial\left[\delta_{g_{j} g} N_{i_{j}, m_{j}} \psi^{(1), g}(r, \boldsymbol{\Omega})\right]}{\partial t_{l}} \\
& =-\psi_{1}^{(2), g}\left(i_{1} ; \boldsymbol{r}, \boldsymbol{\Omega}\right) \frac{\partial \Sigma_{t}^{g}(\boldsymbol{t})}{\partial \sigma_{t, i_{l}}^{g_{l}}}=-\psi_{1}^{(2), g}\left(i_{1} ; \boldsymbol{r}, \boldsymbol{\Omega}\right) \delta_{g_{l} g} N_{i_{l}, m_{l}} .
\end{aligned}
$$


The source $Q_{2}^{(3)}\left[\boldsymbol{\alpha}, \psi_{2}^{(2)}\left(i_{1}\right) ; \delta \boldsymbol{\alpha}\right]$ on the right side in Equation (78) is defined as follows:

$$
Q_{2}^{(3)}\left[\boldsymbol{\alpha}, \psi_{2}^{(2)}\left(i_{1}\right) ; \delta \boldsymbol{\alpha}\right] \triangleq \sum_{i_{3}=1}^{J_{\sigma t}} \frac{\partial Q_{2}^{(3)}\left[\boldsymbol{\alpha}, \psi_{2}^{(2)}\left(i_{1}\right)\right]}{\partial \alpha_{i_{3}}} \delta \alpha_{i_{3}},
$$

where, in view of Equation (67),

$$
\begin{aligned}
& \left\{\frac{\partial Q_{2}^{(3)}\left[\boldsymbol{\alpha}, \psi_{2}^{(2)}\left(i_{1}\right)\right]}{\partial \alpha_{i_{3}}}\right\}_{\alpha_{i_{3}=t_{l}}} \\
& \triangleq-\frac{\partial\left[B^{g}(\boldsymbol{\alpha}) \psi_{2}^{(2), g}\left(i_{1} ; r, \boldsymbol{\Omega}\right)\right]}{\partial t_{l}}-\frac{\partial\left[\delta_{g_{j} g} N_{i_{j}, m_{j}} \varphi^{g}(r, \boldsymbol{\Omega})\right]}{\partial t_{l}} \\
& =-\psi_{2}^{(2), g}\left(i_{1} ; r, \boldsymbol{\Omega}\right) \frac{\partial \Sigma_{t}^{g}(\alpha ; r)}{\partial \sigma_{t, i_{\ell}}^{g_{l}}}=-\psi_{2}^{(2), g}\left(i_{1} ; r, \boldsymbol{\Omega}\right) \delta_{g_{\ell} g} N_{i_{\ell}, m_{\ell}} .
\end{aligned}
$$

The $3^{\text {rd }}$-Level Forward Sensitivity System ( ${ }^{\text {rd }}$-LFSS) comprises Equations (72) through (79). The $3^{\text {rd }}$-Level Adjoint Sensitivity System ( $3^{\text {rd }}$-LASS) that corresponds to the $3^{\text {rd }}$-LFSS is derived by following the general procedure described in Ref. [12], which involves the following sequence of operations:

A. Use the definition of the inner product provided in Equation (15) to perform the following operations:

1) Form the inner product of Equation (72) with a yet undefined vector-valued function

$$
\boldsymbol{\psi}_{1}^{(3), g}(j, k ; r, \mathbf{\Omega}) \triangleq\left[\psi_{1}^{(3), g=1}(j, k ; r, \mathbf{\Omega}), \cdots, \psi_{1}^{(3), g}(j, k ; r, \mathbf{\Omega}), \cdots, \psi_{1}^{(3), g=G}(j, k ; r, \mathbf{\Omega})\right]^{\dagger}
$$

having $G$ square-integrable components $\psi_{1}^{(3), g}(j, k ; r, \Omega)$, to obtain the following relation:

$$
\begin{aligned}
& \left\langle\psi_{1}^{(3), g}(j, k ; r, \boldsymbol{\Omega}), B^{g}(\boldsymbol{\alpha}) \delta \varphi^{g}(r, \boldsymbol{\Omega})\right\rangle_{(1)} \\
& =\left\langle\psi_{1}^{(3), g}(j, k ; r, \boldsymbol{\Omega}), \sum_{l=1}^{J_{\sigma t}}\left[-\delta_{g_{l} g} N_{i_{l}, m_{l}} \varphi^{g}(\boldsymbol{r}, \boldsymbol{\Omega})\right] \delta t_{l}\right\rangle_{(1)} .
\end{aligned}
$$

2) Form the inner product of Equation (74) with a yet undefined vector-valued function

$$
\boldsymbol{\psi}_{2}^{(3), g}(j, k ; r, \mathbf{\Omega}) \triangleq\left[\psi_{2}^{(3), g=1}(j, k ; r, \mathbf{\Omega}), \cdots, \psi_{2}^{(3), g}(j, k ; r, \mathbf{\Omega}), \cdots, \psi_{2}^{(3), g=G}(j, k ; r, \mathbf{\Omega})\right]^{\dagger}
$$

having $G$ square-integrable components $\psi_{2}^{(3), g}(j, k ; r, \Omega)$ to obtain the following relation:

$$
\begin{aligned}
& \left\langle\psi_{2}^{(3), g}(j, k ; r, \boldsymbol{\Omega}), A^{g}(\boldsymbol{\alpha}) \delta \psi^{(1), g}(r, \boldsymbol{\Omega})\right\rangle_{(1)} \\
& =\left\langle\psi_{2}^{(3), g}(j, k ; r, \boldsymbol{\Omega}), \sum_{l=1}^{J_{\sigma t}}\left[-\delta_{g_{l} g} N_{i_{l}, m_{l}} \psi^{(1), g}(\boldsymbol{r}, \boldsymbol{\Omega})\right] \delta t_{l}\right\rangle_{(1)} .
\end{aligned}
$$

3) Form the inner product of Equation (76) with a yet undefined vector-valued function

$\psi_{3}^{(3), g}(j, k ; r, \boldsymbol{\Omega}) \triangleq\left[\psi_{3}^{(3), g=1}(j, k ; r, \boldsymbol{\Omega}), \cdots, \psi_{3}^{(3), g}(j, k ; r, \boldsymbol{\Omega}), \cdots, \psi_{3}^{(3), g=G}(j, k ; r, \boldsymbol{\Omega})\right]^{\dagger}$ 
having $G$ square-integrable components $\psi_{3}^{(3), g}(j, k ; r, \boldsymbol{\Omega})$ to obtain the following relation:

$$
\begin{aligned}
& \left\langle\psi_{3}^{(3), g}(j, k ; r, \boldsymbol{\Omega}), A^{g}(\boldsymbol{\alpha})\left[\delta \psi_{1, j}^{(2), g}(r, \boldsymbol{\Omega})\right]\right\rangle_{(1)} \\
& +\delta_{g_{j} g} N_{i_{j}, m_{j}}\left\langle\psi_{3}^{(3), g}(j, k ; r, \boldsymbol{\Omega}), \delta \psi^{(1), g}(r, \boldsymbol{\Omega})\right\rangle_{(1)} \\
& =\left\langle\psi_{3}^{(3), g}(j, k ; r, \boldsymbol{\Omega}), \sum_{l=1}^{J_{\sigma t}}\left[-\delta_{g_{l} g} N_{i_{l}, m_{l}} \psi_{1, j}^{(2), g}(r, \boldsymbol{\Omega})\right] \delta t_{l}\right\rangle_{(1)} .
\end{aligned}
$$

4) Form the inner product of Equation (78) with a yet undefined vector-valued function

$\boldsymbol{\psi}_{4}^{(3), g}(j, k ; r, \boldsymbol{\Omega}) \triangleq\left[\psi_{4}^{(3), g=1}(j, k ; r, \boldsymbol{\Omega}), \cdots, \psi_{4}^{(3), g}(j, k ; r, \boldsymbol{\Omega}), \cdots, \psi_{4}^{(3), g=G}(j, k ; r, \boldsymbol{\Omega})\right]^{\dagger}$ having $G$ square-integrable components square integrable function $\psi_{4}^{(3), g}(j, k ; r, \boldsymbol{\Omega})$ to obtain the following relation:

$$
\begin{aligned}
& \left\langle\psi_{4}^{(3), g}(j, k ; r, \boldsymbol{\Omega}), B^{g}(\alpha)\left[\delta \psi_{2, j}^{(2), g}(r, \boldsymbol{\Omega})\right]\right\rangle_{(1)} \\
& +\delta_{g_{j} g} N_{i_{j}, m_{j}}\left\langle\psi_{4}^{(3), g}(j, k ; r, \boldsymbol{\Omega}), \delta \varphi^{g}(r, \boldsymbol{\Omega})\right\rangle_{(1)} \\
& =\left\langle\psi_{4}^{(3), g}(j, k ; r, \boldsymbol{\Omega}), \sum_{l=1}^{J_{\sigma t}}\left[-\delta_{g_{l} g} N_{i_{l}, m_{l}} \psi_{2, j}^{(2), g}(r, \boldsymbol{\Omega})\right] \delta t_{l}\right\rangle_{(1)} .
\end{aligned}
$$

5) Add Equations (88) through (91) to obtain the following relation:

$$
\begin{aligned}
& \left\langle\psi_{1}^{(3), g}(j, k ; r, \boldsymbol{\Omega}), B^{g}(\boldsymbol{\alpha}) \delta \varphi^{g}(r, \boldsymbol{\Omega})\right\rangle_{(1)} \\
& +\left\langle\psi_{2}^{(3), g}(j, k ; r, \boldsymbol{\Omega}), A^{g}(\boldsymbol{\alpha}) \delta \psi^{(1), g}(r, \boldsymbol{\Omega})\right\rangle_{(1)} \\
& +\left\langle\psi_{3}^{(3), g}(j, k ; r, \boldsymbol{\Omega}), A^{g}(\boldsymbol{\alpha})\left[\delta \psi_{1, j}^{(2), g}(r, \boldsymbol{\Omega})\right]\right\rangle_{(1)} \\
& +\delta_{g_{j} g} N_{i_{j}, m_{j}}\left\langle\psi_{3}^{(3), g}(j, k ; r, \boldsymbol{\Omega}), \delta \psi^{(1), g}(r, \boldsymbol{\Omega})\right\rangle_{(1)} \\
& +\left\langle\psi_{4}^{(3), g}(j, k ; r, \boldsymbol{\Omega}), B^{g}(\boldsymbol{\alpha})\left[\delta \psi_{2, j}^{(2), g}(r, \boldsymbol{\Omega})\right]\right\rangle_{(1)} \\
& +\delta_{g_{j} g} N_{i_{j}, m_{j}}\left\langle\psi_{4}^{(3), g}(j, k ; r, \boldsymbol{\Omega}), \delta \varphi^{g}(r, \boldsymbol{\Omega})\right\rangle_{(1)} \\
& =\sum_{l=1}^{J_{\sigma t}}\left[-\delta_{g_{l} g} N_{i_{l}, m_{l}}\left\langle\psi_{1}^{(3), g}(j, k ; r, \boldsymbol{\Omega}), \varphi^{g}(r, \boldsymbol{\Omega})\right\rangle_{(1)}\right. \\
& -\delta_{g_{l} g} N_{i_{i}, m_{l}}\left\langle\psi_{2}^{(3), g}(j, k ; r, \boldsymbol{\Omega}), \psi^{(1), g}(r, \boldsymbol{\Omega})\right\rangle_{(1)} \\
& -\delta_{g_{l} g} N_{i_{l}, m_{l}}\left\langle\psi_{3}^{(3), g}(j, k ; r, \boldsymbol{\Omega}), \psi_{1, j}^{(2), g}(r, \boldsymbol{\Omega})\right\rangle_{(1)} \\
& \left.-\delta_{g_{l} g} N_{i_{l}, m_{l}}\left\langle\psi_{4}^{(3), g}(j, k ; r, \boldsymbol{\Omega}), \psi_{2, j}^{(2), g}(r, \boldsymbol{\Omega})\right\rangle_{(1)}\right] \delta t_{l} .
\end{aligned}
$$

B. Use the relations between the forward and adjoint multigroup transport operators to recast the left side of Equation (92) into the following form:

$$
\begin{aligned}
& \left\langle\psi_{1}^{(3), g}(j, k ; r, \boldsymbol{\Omega}), B^{g}(\boldsymbol{\alpha}) \delta \varphi^{g}(r, \boldsymbol{\Omega})\right\rangle_{(1)} \\
& +\left\langle\psi_{2}^{(3), g}(j, k ; r, \boldsymbol{\Omega}), A^{g}(\boldsymbol{\alpha}) \delta \psi^{(1), g}(r, \boldsymbol{\Omega})\right\rangle_{(1)}
\end{aligned}
$$




$$
\begin{aligned}
& \left\langle\psi_{3}^{(3), g}(j, k ; r, \boldsymbol{\Omega}), A^{g}(\boldsymbol{\alpha}) \delta \psi_{1, j}^{(2), g}(r, \boldsymbol{\Omega})\right\rangle_{(1)} \\
+ & \delta_{g_{j} g} N_{i_{j}, m_{j}}\left\langle\psi_{3}^{(3), g}(j, k ; r, \boldsymbol{\Omega}), \delta \psi^{(1), g}(r, \boldsymbol{\Omega})\right\rangle_{(1)} \\
+ & \left\langle\psi_{4}^{(3), g}(j, k ; r, \boldsymbol{\Omega}), B^{g}(\boldsymbol{\alpha}) \delta \psi_{2, j}^{(2), g}(r, \boldsymbol{\Omega})\right\rangle_{(1)} \\
& +\delta_{g_{j} g} N_{i_{j}, m_{j}}\left\langle\psi_{4}^{(3), g}(j, k ; r, \boldsymbol{\Omega}), \delta \varphi^{g}(r, \boldsymbol{\Omega})\right\rangle_{(1)} \\
= & \left\langle\delta \varphi^{g}(r, \boldsymbol{\Omega}), A^{g}(\alpha) \psi_{1}^{(3), g}(j, k ; r, \boldsymbol{\Omega})+\delta_{g_{j} g} N_{i_{j}, m_{j}} \psi_{4}^{(3), g}(j, k ; r, \boldsymbol{\Omega})\right\rangle_{(1)} \\
& +\left\langle\delta \psi^{(1), g}(r, \boldsymbol{\Omega}), B^{g}(\alpha) \psi_{2}^{(3), g}(j, k ; r, \boldsymbol{\Omega})+\delta_{g_{j} g} N_{i_{j}, m_{j}} \psi_{3}^{(3), g}(j, k ; r, \boldsymbol{\Omega})\right\rangle_{(1)} \\
+ & \left\langle\delta \psi_{1, j}^{(2), g}(r, \boldsymbol{\Omega}), B^{g}(\alpha) \psi_{3}^{(3), g}(j, k ; r, \boldsymbol{\Omega})\right\rangle_{(1)} \\
+ & \left\langle\delta \psi_{2, j}^{(2), g}(r, \boldsymbol{\Omega}), A^{g}(\boldsymbol{\alpha}) \psi_{4}^{(3), g}(j, k ; r, \boldsymbol{\Omega})\right\rangle_{(1)} \\
+ & P^{(3)}\left[\delta \varphi^{g}, \delta \psi^{(1), g} ; \delta \psi_{1}^{(2), g}\left(i_{1}\right), \delta \psi_{2}^{(2), g}\left(i_{1}\right) ;\right. \\
& \left.\psi_{1}^{(3), g}\left(i_{1}, i_{2}\right), \psi_{2}^{(3), g}\left(i_{1}, i_{2}\right), \psi_{3}^{(3), g}\left(i_{1}, i_{2}\right), \psi_{4}^{(3), g}\left(i_{1}, i_{2}\right)\right]
\end{aligned}
$$

where $P^{(3)}$ denotes the corresponding bilinear concomitant on the domain's boundary in the phase-space of independent variables.

C. Use the boundary conditions provided in Equations (73), (75), (77) and (79), and impose on the functions $\psi_{m}^{(3), g}(j, k ; r, \Omega), m=1,2,3,4$, the boundary conditions provided in Equations (95), (97), (99) and (101), below, in order to cause the bilinear concomitant $P^{(3)}$ in Equation (93) to vanish.

D. Identify the right side of Equation (93) with the right side of Equations (71) to obtain the following equations for the $3^{\text {rd }}$-LASS, where

$$
\begin{gathered}
j=1, \cdots, J_{\sigma t} ; k=1, \cdots, j ; g=1, \cdots, G: \\
A^{g}(\alpha) \psi_{1}^{(3), g}(j, k ; r, \boldsymbol{\Omega})+\delta_{g_{j} g} N_{i_{j}, m_{j}} \psi_{4}^{(3), g}(j, k ; r, \boldsymbol{\Omega})=-\delta_{g_{k} g} N_{i_{k}, m_{k}} \psi_{1, j}^{(2), g}(r, \boldsymbol{\Omega}), \\
\psi_{1}^{(3), g}\left(j, k ; r_{d}, \boldsymbol{\Omega}\right)=0, \boldsymbol{\Omega} \cdot \boldsymbol{n}>0, \quad j=1, \cdots, J_{\sigma t}, k=1, \cdots, j, g=1, \cdots, G, \\
B^{g}(\boldsymbol{\alpha}) \psi_{2}^{(3), g}(j, k ; r, \boldsymbol{\Omega})+\delta_{g_{j} g} N_{i_{j}, m_{j}} \psi_{3}^{(3), g}(j, k ; r, \boldsymbol{\Omega})=-\delta_{g_{k} g} N_{i_{k}, m_{k}} \psi_{2, j}^{(2), g}(r, \boldsymbol{\Omega}), \\
\psi_{2}^{(3), g}\left(j, k ; r_{d}, \boldsymbol{\Omega}\right)=0, \boldsymbol{\Omega} \cdot \boldsymbol{n}<0, j=1, \cdots, J_{\sigma t}, k=1, \cdots, j, g=1, \cdots, G, \\
B^{g}(\boldsymbol{\alpha}) \psi_{3}^{(3), g}(j, k ; r, \boldsymbol{\Omega})=-\delta_{g_{k} g} N_{i_{k}, m_{k}} \varphi^{g}(r, \boldsymbol{\Omega}), \\
\psi_{3}^{(3), g}\left(j, k ; r_{d}, \boldsymbol{\Omega}\right)=0, \boldsymbol{\Omega} \cdot \boldsymbol{n}<0, j=1, \cdots, J_{\sigma t}, k=1, \cdots, j, g=1, \cdots, G, \\
A^{g}(\boldsymbol{\alpha}) \psi_{4}^{(3), g}(j, k ; r, \boldsymbol{\Omega})=-\delta_{g_{k} g} N_{i_{k}, m_{k}} \psi^{(1), g}(r, \boldsymbol{\Omega}), \\
\psi_{4}^{(3), g}\left(j, k ; r_{d}, \boldsymbol{\Omega}\right)=0, \boldsymbol{\Omega} \cdot \boldsymbol{n}>0, j=1, \cdots, J_{\sigma t}, k=1, \cdots, j, g=1, \cdots, G .
\end{gathered}
$$

E. It follows from the $3^{\text {rd }}$-LASS defined in Equations (94) through (101) that 
the right-sides of Equations (92) and (71) represent the same functional, which implies that

$$
\begin{aligned}
& \frac{\partial^{3} L(\boldsymbol{\alpha})}{\partial t_{j} \partial t_{k} \partial t_{\ell}}=-\delta_{g_{j} g} N_{i_{j}, m_{j}}\left\{\left\langle\psi_{1}^{(3), g}(j, k ; r, \boldsymbol{\Omega}), \varphi^{g}(r, \boldsymbol{\Omega})\right\rangle_{(1)}\right. \\
&+\left\langle\psi_{2}^{(3), g}(j, k ; r, \boldsymbol{\Omega}), \psi^{(1), g}(r, \boldsymbol{\Omega})\right\rangle_{(1)} \\
&+\left\langle\psi_{3}^{(3), g}(j, k ; r, \boldsymbol{\Omega}), \psi_{1, j}^{(2), g}(r, \boldsymbol{\Omega})\right\rangle_{(1)} \\
&\left.+\left\langle\psi_{4}^{(3), g}(j, k ; r, \boldsymbol{\Omega}), \psi_{2, j}^{(2), g}(r, \boldsymbol{\Omega})\right\rangle_{(1)}\right\}, \\
& \text { for } j=1, \cdots, J_{\sigma t}, k=1, \cdots, j, \quad \ell=1, \cdots, k .
\end{aligned}
$$

As expected, the $3^{\text {rd }}$-LASS obtained in Equations (94) through (101) is identical to the $3^{\text {rd }}$-LASS obtained in Equations (43) through (50), and the expression obtained for the $3^{\text {rd }}$-order sensitivities $\partial^{3} L(\alpha) / \partial t_{j} \partial t_{k} \partial t_{\ell}, j, k, \ell=1, \cdots, J_{\sigma t}$ obtained in Equation (102) is identical to the expression obtained in Equation (69).

\section{Concluding Remarks}

This work has presented the derivation of the exact mathematical expressions of the $(180)^{3}$ third-order sensitivities of the PERP leakage response with respect to the total microscopic cross sections. By using the solution of the $3^{\text {rd }}$-Level Adjoint Sensitivity System, the expressions of these $3^{\text {rd }}$-order sensitivities can be computed selectively and most efficiently. The formulas derived in this work are valid not only for the PERP benchmark but can also be used for computing the $3^{\text {rd }}$-order sensitivities of the leakage response of any nuclear system involving fissionable material and internal or external neutron sources. Subsequent works will use the adjoint-based mathematical expressions obtained in this work to compute exactly and efficiently the numerical values of these $(180)^{3}$ third-order sensitivities (which turned out to be very large and consequential) and use them for a third-order uncertainty analysis of the PERP benchmark's leakage response.

\section{Conflicts of Interest}

The authors declare no conflicts of interest regarding the publication of this paper.

\section{References}

[1] Cacuci, D.G. (2018) The Second-Order Adjoint Sensitivity Analysis Methodology. CRC Press, New York. https://doi.org/10.1201/9781315120270

[2] Cacuci, D.G. (2019) Application of the Second-Order Comprehensive Adjoint Sensitivity Analysis Methodology to Compute 1st- and 2nd-Order Sensitivities of Flux Functionals in a Multiplying System with Source. Nuclear Science and Engineering 193, 555-600. https://doi.org/10.1080/00295639.2018.1553910

[3] Cacuci, D.G. (2019) Second-Order Sensitivities of a General Functional of the Forward and Adjoint Fluxes in a Multiplying Nuclear System with Source. Nuclear Engineering and Design, 344, 83-106. https://doi.org/10.1016/j.nucengdes.2019.01.007 
[4] Cacuci, D.G. (2020) Second-Order Adjoint Sensitivity Analysis of a General Ratio of Functionals of the Forward and Adjoint Fluxes in a Multiplying Nuclear System with Source. Annals of Nuclear Energy, 135, Article ID: 106956. https://doi.org/10.1016/j.anucene.2019.106956

[5] Cacuci, D.G., Fang, R. and Favorite, J.A. (2019) Comprehensive Second-Order Adjoint Sensitivity Analysis Methodology (2nd-ASAM) Applied to a Subcritical Experimental Reactor Physics Benchmark: I. Effects of Imprecisely Known Microscopic Total and Capture Cross Sections. Energies, 12, 4219.

https://doi.org/10.3390/en12214219

[6] Fang, R. and Cacuci, D.G. (2019) Comprehensive Second-Order Adjoint Sensitivity Analysis Methodology (2nd-ASAM) Applied to a Subcritical Experimental Reactor Physics Benchmark: II. Effects of Imprecisely Known Microscopic Scattering Cross Sections. Energies, 12, 4114. https://doi.org/10.3390/en12214114

[7] Cacuci, D.G., Fang, R., Favorite, J.A., Badea, M.C. and di Rocco, F. (2019) Comprehensive Second-Order Adjoint Sensitivity Analysis Methodology (2nd-ASAM) Applied to a Subcritical Experimental Reactor Physics Benchmark: III. Effects of Imprecisely Known Microscopic Fission Cross Sections and Average Number of Neutrons Per Fission. Energies, 12, 4100. https://doi.org/10.3390/en12214100

[8] Fang, R. and Cacuci, D.G. (2020) Comprehensive Second-Order Adjoint Sensitivity Analysis Methodology (2nd-ASAM) Applied to a Subcritical Experimental Reactor Physics Benchmark: IV. Effects of Imprecisely Known Source Parameters. Energies, 13, 1431. https://doi.org/10.3390/en13061431

[9] Fang, R. and Cacuci, D.G. (2020) Comprehensive Second-Order Adjoint Sensitivity Analysis Methodology (2nd-ASAM) Applied to a Subcritical Experimental Reactor Physics Benchmark: V. Computation of Mixed 2nd-Order Sensitivities Involving Isotopic Number Densities. Energies, 13, 2580. https://doi.org/10.3390/en13102580

[10] Cacuci, D.G., Fang, R. and Favorite, J.A. (2020) Comprehensive Second-Order Adjoint Sensitivity Analysis Methodology (2nd-ASAM) Applied to a Subcritical Experimental Reactor Physics Benchmark: VI. Overall Impact of 1st- and 2nd-Order Sensitivities on Response Uncertainties. Energies, 13, 1674,

https://doi.org/10.3390/en13071674

[11] Valentine, T.E. (2006) Polyethylene-Reflected Plutonium Metal Sphere Subcritical Noise Measurements, SUB-PU-METMIXED-001. International Handbook of Evaluated Criticality Safety Benchmark Experiments, NEA/NSC/DOC(95)03/I-IX, Organization for Economic Co-Operation and Development, Nuclear Energy Agency, Paris.

[12] Cacuci, D.G. (2021) Third Order Adjoint Sensitivity Analysis of Reaction Rate Responses in a Multiplying Nuclear System with Source. Annals of Nuclear Energy, 151, Article ID: 107924. https://doi.org/10.1016/j.anucene.2020.107924

[13] Fang, R. and Cacuci, D.G. (2020) Third Order Adjoint Sensitivity and Uncertainty Analysis of an OECD/NEA Reactor Physics Benchmark: II. Computed Sensitivities. American Journal of Computational Mathematics, 10, 529-558. https://doi.org/10.4236/ajcm.2020.104030

[14] Fang, R. and Cacuci, D.G. (2020) Third Order Adjoint Sensitivity and Uncertainty Analysis of an OECD/NEA Reactor Physics Benchmark: III. Response Moments. American Journal of Computational Mathematics, 10, 559-570. https://doi.org/10.4236/ajcm.2020.104031

[15] Alcouffe, R.E., Baker, R.S., Dahl, J.A., Turner, S.A. and Ward, R. (2008) PARTISN: A 
Time-Dependent, Parallel Neutral Particle Transport Code System. LA-UR-08-07258, Los Alamos National Laboratory, Los Alamos.

[16] Wilson, W.B., Perry, R.T., Shores, E.F., Charlton, W.S., Parish, T.A., Estes, G.P., Brown, T.H., Arthur, E.D., Bozoian, M., England, T.R., et al. (2002) SOURCES4C: A Code for Calculating $(\alpha, n)$, Spontaneous Fission, and Delayed Neutron Sources and Spectra. Proceedings of the American Nuclear Societyl Radiation Protection and Shielding Division 12 th Biennial Topical Meeting, Santa Fe, 14-18 April 2002, 1-127.

[17] Conlin, J.L., Parsons, D.K., Gardiner, S.J., Beth Lee, M.G.M. and White, M.C. (2013) MENDF71X: Multigroup Neutron Cross-Section Data Tables Based upon ENDF/B-VII.1X. Report LA-UR-15-29571, Los Alamos National Laboratory, Los Alamos, 1-11. 


\section{Appendix}

The dimensions and material composition of the polyethylene-reflected plutonium (PERP) metal sphere considered in this work are presented in Table A1.

The scattering cross section $\sum_{s}^{g^{\prime} \rightarrow g}\left(r, \mathbf{\Omega}^{\prime} \rightarrow \mathbf{\Omega}\right) \rightarrow \Sigma_{s}^{g^{\prime} \rightarrow g}\left(s ; r, \mathbf{\Omega}^{\prime} \rightarrow \mathbf{\Omega}\right)$ will be characterized by the vector of parameters $s$, which is defined as follows:

$$
\boldsymbol{s} \triangleq\left[s_{1}, \cdots, s_{J_{s}}\right]^{\dagger} \triangleq\left[s_{1}, \cdots, s_{J_{\sigma s}} ; n_{1}, \cdots, n_{J_{n}}\right]^{\dagger} \triangleq\left[\sigma_{s} ; \boldsymbol{N}\right]^{\dagger}, J_{s} \triangleq J_{\sigma s}+J_{n},
$$

Table A1. Dimensions and material composition of the PERP benchmark.

\begin{tabular}{|c|c|c|c|c|}
\hline Materials & Isotopes & Weight Fraction & Density $\left(\mathrm{g} / \mathrm{cm}^{3}\right)$ & Zones \\
\hline \multirow{5}{*}{$\begin{array}{l}\text { Material } 1 \\
\text { (plutonium } \\
\text { metal) }\end{array}$} & Isotope $1\left({ }^{239} \mathrm{Pu}\right)$ & $9.3804 \times 10^{-1}$ & \multirow{5}{*}{19.6} & \multirow{5}{*}{$\begin{array}{l}\text { Homogeneous sphere } \\
\text { of radius } r_{1}=3.794 \mathrm{~cm} \text {, } \\
\text { designated as "material 1" } \\
\text { and assigned to zone } 1\end{array}$} \\
\hline & & & & \\
\hline & Isotope $2\left({ }^{240} \mathrm{Pu}\right)$ & $5.9411 \times 10^{-2}$ & & \\
\hline & Isotope $3\left({ }^{69} \mathrm{Ga}\right)$ & $1.5152 \times 10^{-3}$ & & \\
\hline & Isotope $4\left({ }^{71} \mathrm{Ga}\right)$ & $1.0346 \times 10^{-3}$ & & \\
\hline \multirow[b]{2}{*}{$\begin{array}{l}\text { Material } 2 \\
\text { (polyethylene) }\end{array}$} & Isotope $5(\mathrm{C})$ & $8.5630 \times 10^{-1}$ & \multirow[b]{2}{*}{0.95} & $\begin{array}{l}\text { Homogeneous spherical } \\
\text { shell of inner radius }\end{array}$ \\
\hline & Isotope $6\left({ }^{1} \mathrm{H}\right)$ & $1.4370 \times 10^{-1}$ & & $\begin{array}{l}r_{1}=3.794 \mathrm{~cm} \text { and outer } \\
\text { radius } r_{2}=7.604 \mathrm{~cm}, \\
\text { designated as "material 2" } \\
\text { and assigned to zone } 2\end{array}$ \\
\hline
\end{tabular}

Table A2. Presents the group boundaries, $E^{g}$, of the $G=30$ energy groups used in the PARTISN forward and adjoint neutron transport computations.

\begin{tabular}{|c|c|c|c|c|c|c|}
\hline$g$ & 1 & 2 & 3 & 4 & 5 & 6 \\
\hline$E^{g}$ & $1.50 \times 10^{1}$ & $1.35 \times 10^{1}$ & $1.20 \times 10^{1}$ & $1.00 \times 10^{1}$ & $7.79 \times 10^{0}$ & $6.07 \times 10^{0}$ \\
\hline$E^{g-1}$ & $1.70 \times 10^{1}$ & $1.50 \times 10^{1}$ & $1.35 \times 10^{1}$ & $1.20 \times 10^{1}$ & $1.00 \times 10^{1}$ & $7.79 \times 10^{0}$ \\
\hline$g$ & 7 & 8 & 9 & 10 & 11 & 12 \\
\hline$E^{g}$ & $3.68 \times 10^{0}$ & $2.87 \times 10^{0}$ & $2.23 \times 10^{0}$ & $1.74 \times 10^{0}$ & $1.35 \times 10^{0}$ & $8.23 \times 10^{-1}$ \\
\hline$E^{g-1}$ & $6.07 \times 10^{0}$ & $3.68 \times 10^{0}$ & $2.87 \times 10^{0}$ & $2.23 \times 10^{0}$ & $1.74 \times 10^{0}$ & $1.35 \times 10^{0}$ \\
\hline$g$ & 13 & 14 & 15 & 16 & 17 & 18 \\
\hline$E^{g}$ & $5.00 \times 10^{-1}$ & $3.03 \times 10^{-1}$ & $1.84 \times 10^{-1}$ & $6.76 \times 10^{-2}$ & $2.48 \times 10^{-2}$ & $9.12 \times 10^{-3}$ \\
\hline$E^{g-1}$ & $8.23 \times 10^{-1}$ & $5.00 \times 10^{-1}$ & $3.03 \times 10^{-1}$ & $1.84 \times 10^{-1}$ & $6.76 \times 10^{-2}$ & $2.48 \times 10^{-2}$ \\
\hline$g$ & 19 & 20 & 21 & 22 & 23 & 24 \\
\hline$E^{g}$ & $3.35 \times 10^{-3}$ & $1.24 \times 10^{-3}$ & $4.54 \times 10^{-4}$ & $1.67 \times 10^{-4}$ & $6.14 \times 10^{-5}$ & $2.26 \times 10^{-5}$ \\
\hline$E^{g-1}$ & $9.12 \times 10^{-3}$ & $3.35 \times 10^{-3}$ & $1.24 \times 10^{-3}$ & $4.54 \times 10^{-4}$ & $1.67 \times 10^{-4}$ & $6.14 \times 10^{-5}$ \\
\hline$g$ & 25 & 26 & 27 & 28 & 29 & 30 \\
\hline$E^{g}$ & $8.32 \times 10^{-6}$ & $3.06 \times 10^{-6}$ & $1.13 \times 10^{-6}$ & $4.14 \times 10^{-7}$ & $1.52 \times 10^{-7}$ & $1.39 \times 10^{-10}$ \\
\hline$E^{g-1}$ & $2.26 \times 10^{-5}$ & $8.32 \times 10^{-6}$ & $3.06 \times 10^{-6}$ & $1.13 \times 10^{-6}$ & $4.14 \times 10^{-7}$ & $1.52 \times 10^{-7}$ \\
\hline
\end{tabular}


where

$$
\begin{aligned}
\sigma_{s} \triangleq & {\left[s_{1}, \cdots, s_{J_{\sigma s}}\right]^{\dagger} } \\
\triangleq & {\left[\sigma_{s, l=0, i=1}^{g^{\prime}=1 \rightarrow g=1}, \sigma_{s, l=0, i=1}^{g^{\prime}=2 \rightarrow g=1}, \cdots, \sigma_{s, l=0, i=1}^{g^{\prime}=G \rightarrow g=1}, \sigma_{s, l=0, i=1}^{g^{\prime}=1 \rightarrow g=2},\right.} \\
& \left.\sigma_{s, l=0, i=1}^{g^{\prime}=2 \rightarrow g=2}, \cdots, \sigma_{s, l, i}^{g^{\prime} \rightarrow g}, \cdots, \sigma_{s, I S C T, i=I}^{G \rightarrow G}\right]^{\dagger}, \\
l= & 0, \cdots, I S C T ; i=1, \cdots, I ; g, g^{\prime}=1, \cdots, G ; \quad J_{\sigma s}=(G \times G) \times I \times(I S C T+1) .
\end{aligned}
$$

The quantity $\left(v \Sigma_{f}\right)^{g} \rightarrow\left(v \Sigma_{f}\right)^{g}(\boldsymbol{f} ; r)$ in the fission integral $\int(v \Sigma)_{f}^{g^{\prime}}(r) \varphi^{g^{\prime}}\left(r, \boldsymbol{\Omega}^{\prime}\right) \mathrm{d} \boldsymbol{\Omega}^{\prime}$ depends on the vector of parameters $\boldsymbol{f}$, which is deffined as follows:

$$
\begin{aligned}
\boldsymbol{f} & \triangleq\left[f_{1}, \cdots, f_{J_{\sigma f}} ; f_{J_{\sigma f}+1}, \cdots, f_{J_{\sigma f}+J_{v}} ; f_{J_{\sigma f}+J_{v}+1}, \cdots, f_{J_{f}}\right]^{\dagger} \\
& \triangleq\left[\boldsymbol{\sigma}_{f} ; \boldsymbol{v} ; \boldsymbol{N}\right]^{\dagger}, J_{f}=J_{\sigma f}+J_{v}+J_{n},
\end{aligned}
$$

with

$$
\begin{gathered}
\sigma_{f} \triangleq\left[\sigma_{f, i=1}^{1}, \sigma_{f, i=1}^{2}, \cdots, \sigma_{f, i=1}^{G}, \cdots, \sigma_{f, i}^{g}, \cdots, \sigma_{f, i=N_{f}}^{1}, \cdots, \sigma_{f, i=N_{f}}^{G}\right]^{\dagger} \\
\triangleq\left[f_{1}, \cdots, f_{J_{\sigma f}}\right]^{\dagger}, i=1, \cdots, N_{f} ; g=1, \cdots, G ; J_{\sigma f}=G \times N_{f}, \\
v \triangleq\left[v_{i=1}^{1}, v_{i=1}^{2}, \cdots, v_{i=1}^{G}, \cdots, v_{i}^{g}, \cdots, v_{i=N_{f}}^{1}, \cdots, v_{i=N_{f}}^{G}\right]^{\dagger} \triangleq\left[f_{J_{\sigma f}+1}, \cdots, f_{J_{\sigma f}+J_{v}}\right]^{\dagger}, \\
i=1, \cdots, N_{f} ; g=1, \cdots, G ; J_{v}=G \times N_{f},
\end{gathered}
$$

where $\sigma_{f, i}^{g}$ denotes the microscopic fission cross section for isotope $i$ and energy group $g, v_{i}^{g}$ denotes the average number of neutrons per fission for isotope $i$ and energy group $g$, and $N_{f}$ denotes the total number of fissionable isotopes.

The fission spectrum is considered to depend on the vector of parameters $\boldsymbol{p}$, defined as follows:

$$
\begin{aligned}
& \boldsymbol{p} \triangleq\left[p_{1}, \cdots, p_{J_{p}}\right]^{\dagger} \triangleq\left[\chi_{i=1}^{g=1}, \chi_{i=1}^{g=2}, \cdots, \chi_{i=1}^{G}, \cdots, \chi_{i}^{g}, \cdots, \chi_{N_{f}}^{G}\right]^{\dagger}, \\
& \quad \text { for } i=1, \cdots, N_{f} ; g=1, \cdots, G ; \quad J_{p}=G \times N_{f} .
\end{aligned}
$$

The quantities $\chi^{g}(r)$ further depend on the parameters $\chi_{i}^{g}, N_{i, m}, f_{i}^{g}$, $\left(v \sigma_{f}\right)_{i}^{g}$, but these latter dependences can be taken into account by applying the chain rule on the $1^{\text {st }}$-order sensitivities $\partial L / \partial \chi^{g}$, once these sensitivities will have been obtained.

The source $Q^{g}(r) \rightarrow Q^{g}(\boldsymbol{q} ; \boldsymbol{N} ; r)$ depends on the vector of model parameters $\boldsymbol{q}$, defined as follows:

$$
\boldsymbol{q} \triangleq\left[q_{1}, \cdots, q_{J_{q}}\right]^{\dagger} \triangleq\left[\lambda_{1}, \lambda_{2} ; F_{1}^{S F}, F_{2}^{S F} ; a_{1}, a_{2} ; b_{1}, b_{2} ; v_{1}^{S F}, v_{2}^{S F}\right]^{\dagger}, J_{q}=10 .
$$

In summary, the model parameters characterizing the PERP benchmark can all be considered to be the components of the following "vector of model parameters:"

$$
\begin{aligned}
& \boldsymbol{\alpha} \triangleq\left[\alpha_{1}, \cdots, \alpha_{J_{\alpha}}\right]^{\dagger} \triangleq\left[\boldsymbol{\sigma}_{t} ; \boldsymbol{\sigma}_{s} ; \boldsymbol{\sigma}_{f} ; \boldsymbol{v} ; \boldsymbol{p} ; \boldsymbol{q} ; \boldsymbol{N}\right]^{\dagger}, \\
& J_{\alpha}=J_{\sigma t}+J_{\sigma s}+J_{\sigma f}+J_{v}+J_{p}+J_{q}+J_{n} .
\end{aligned}
$$


Thus, the total number of imprecisely known model parameters for the PERP benchmark is:

$$
J_{\alpha}=(I \times G)+(G \times G) \times I \times(I S C T+1)+2\left(G \times N_{f}\right)+G \times N_{f}+10+J_{n}=21976 .
$$

\section{Nomenclature}

$a_{k}, \quad b_{k}$ : parameters used in Watt's fission spectra approximation for isotope $k$ $B$ : forward Boltzmann operator

$E^{g}$ : boundary of energy group $g$

$F_{k}^{S F}$ : fraction of isotope $k$ decays that are spontaneous fission events

$f_{j}, f_{m_{2}}$ : parameters in vector $\sigma_{f}$ indexed by $j$ and $m_{2}$

$G$ : total number of energy groups

I: total number of isotopes

$J_{n}$ : total number of parameters in vector $N$

$J_{p}$ : total number of parameters in vector $\boldsymbol{p}$

$J_{q}:$ total number of parameters in vector $\boldsymbol{q}$

$J_{\sigma f}$ : total number of parameters in vector $\sigma_{f}$

$J_{\sigma s}$ : total number of parameters in vector $\sigma_{s}$

$J_{\sigma t}$ : total number of parameters in vector $\sigma_{t}$

$J_{t}$ : total number of parameters in vector $\boldsymbol{t}$

$J_{v}$ : total number of parameters in vector $v$

$l$ : variable for the order of Legendre-expansion of the microscopic scattering cross sections, $l=1, \cdots, I S C T$

$L(\alpha)$ : total neutron leakage from the PERP sphere

$M$ : total number of materials

$N_{f}$ : total number of fissionable isotopes

$N_{i, m}$ : atom number density for isotope $i$ and material $m$

$P_{l}\left(\boldsymbol{\Omega}^{\prime} \cdot \mathbf{\Omega}\right)$ : Legendre and associated Legendre polynomials appreciate for the geometry

$P_{l}(\mu)$ : spherical harmonics appreciate for the geometry

$Q^{g}(r)$ : source term in group $g$

$r$ spatial variable

$r_{d}$ : external radius of the PERP benchmark

$S_{b}$ : outer surface of the PERP sphere

$s_{f, i}^{g}$ : standard deviation associated with the model parameter $\sigma_{f, i}^{g}$

$s_{v, i}^{g}:$ standard deviation associated with the model parameter $v_{i}^{g}$

$s_{j}, s_{m_{2}}$ : parameters in vector $\sigma_{s}$ indexed by $j$ and $m_{2}$

$t_{j}, t_{m_{2}}$ : parameters in vector $\sigma_{t}$ indexed by $j$ and $m_{2}$

\section{Vectors and Matrices}

$\alpha$ : vector of imprecisely known model parameters, $\alpha \triangleq\left[\sigma_{t} ; \boldsymbol{\sigma}_{s} ; \boldsymbol{\sigma}_{f} ; \boldsymbol{v} ; \boldsymbol{p} ; \boldsymbol{q} ; \boldsymbol{N}\right]^{\dagger}$

$\alpha^{0}:$ nominal values of the parameters in the vector $\alpha$

$\boldsymbol{t}$ : vector of imprecisely known total parameters, $t \triangleq\left[\sigma_{t} ; N\right]^{\dagger}$ 
$s$ : vector of imprecisely known scatter parameters, $s \triangleq\left[\sigma_{s} ; N\right]^{\dagger}$

$f$ : vector of imprecisely known fission parameters, $f \triangleq\left[\sigma_{f} ; \boldsymbol{v} ; N\right]^{\dagger}$

$\sigma_{t}$ : vector of imprecisely known total cross sections

$\sigma_{s}$ : vector of imprecisely known scattering cross sections

$\sigma_{f}$ : vector of imprecisely known fission cross sections

$v$ : vector of imprecisely known parameters underlying the average number of neutrons per fission

$N$ : vector of imprecisely known atom number densities

$\boldsymbol{p}$ : vector of imprecisely known fission spectrum parameters

$\boldsymbol{q}$ : vector of imprecisely known source parameters

$\boldsymbol{S}^{(1)}$ : vector of first-order relative sensitivities of the leakage response

$S^{(2)}$ : matrix of first-order relative sensitivities of the leakage response

$\lambda_{k}$ : decay constant for isotope $k$

$v_{k}^{S F}$ : the spontaneous emission of an average neutrons of an isotope $k$

$\sigma_{f, i}^{g}$ : microscopic fission cross section in group $g$ of isotope $i$

$\sigma_{s, l, i}^{g^{\prime} \rightarrow g}:$ the $l^{\text {th }}$ order Legendre-expanded microscopic scattering cross section from energy group $g^{\prime}$ into energy group $g$ for isotope $i$

$\sigma_{t, i}^{g}:$ microscopic total cross section in group $g$ of isotope $i$

$\Sigma_{t}^{g}(\boldsymbol{t} ; r)$ : macroscopic total cross section for energy group $g$

$\Sigma_{f}^{g}(f ; r):$ macroscopic fission cross section for energy group $g$

$\Sigma_{s}^{g^{\prime} \rightarrow g}\left(s ; r, \boldsymbol{\Omega}^{\prime} \rightarrow \boldsymbol{\Omega}\right)$ : macroscopic scattering transfer cross section from energy

group $g^{\prime}$ into energy group $g$

$\varphi^{g}(r, \boldsymbol{\Omega})$ : forward angular flux in group $g$ at point $r$ in direction $\mathbf{\Omega}$

$\varphi_{0}^{g}(r)$ : zeroth order of forward flux moment in group $g$ at point $r$

$\varphi_{l}^{g}(r): l^{\text {th }}(l=1, \cdots, I S C T)$ order forward flux moment in group $g$ at point $r$,

$\varphi_{l}^{g}(r) \triangleq \int_{4 \pi} \mathrm{d} \boldsymbol{\Omega} P_{l}(\mu) \varphi^{g}(r, \boldsymbol{\Omega}), l=1, \cdots, I S C T$

$\chi^{g}(r)$ : material fission spectrum in energy group $g$

$\psi^{(1), g}(r, \Omega)$ : adjoint angular flux in group $g$ at point $r$ in direction $\Omega$

$\boldsymbol{\Omega}, \boldsymbol{\Omega}^{\prime}$ : directional variable

\section{Subscripts, Superscripts}

\section{f. fission}

$g, g^{\prime}$ : energy group variable $g, g^{\prime}=1, \cdots, G$

$g_{j}, g_{m_{2}}$ : energy group associated with parameter indexed by $j$ (e.g., $f_{j}, t_{j}$ and $s_{j}$ ) or $m_{2}$ (e.g., $f_{m_{2}}, t_{m_{2}}$ and $s_{m_{2}}$ )

$i$ : index variable for isotopes, $i=1, \cdots, I$

$i_{j}, i_{m_{2}}$ : isotope associated with the parameter indexed by $j$ (e.g., $f_{j}, t_{j}$ and $s_{j}$ ) or $m_{2}$ (e.g., $f_{m_{2}}, t_{m_{2}}$ and $s_{m_{2}}$ )

$j:$ index variable for parameters

$k$. index variable for isotopes, $k=1, \cdots, I$

$l_{j}, l_{m_{2}}$ : order of Legendre expansion associated with the microscopic scattering cross section parameters indexed by $j\left(\right.$ e.g., $\left.s_{j}\right)$ or $m_{2}$ (e.g., $s_{m_{2}}$ ) 
$v$ : number of neutrons produced per fission

$m$ : index variable for materials, $m=1, \cdots, M$

$m_{2}:$ index variable for parameters

$m_{j}, m_{m_{2}}$ : material associated with parameter indexed by $j$ (e.g., $f_{j}, t_{j}$ and $s_{j}$ ) or $m_{2}$ (e.g., $f_{m_{2}}, t_{m_{2}}$ and $s_{m_{2}}$ )

t. total

s. scatter

\section{Abbreviations}

$1^{\text {st }}$-LASS: $1^{\text {st }}$-Level adjoint sensitivity system

$2^{\text {nd }}-A S A M$ : second-order adjoint sensitivity analysis methodology

$2^{\text {nd }}$-CASAM: comprehensive second-order adjoint sensitivity analysis methodology

$2^{\text {nd }}$-LASS: $2^{\text {nd }}-$ Level adjoint sensitivity system

$3^{\text {rd }}$-LASS: $3^{\text {rd }}$-Level adjoint sensitivity system

$\mathrm{Nu}$ : the average number of neutrons per fission

ISCT: order of the finite expansion in Legendre polynomial

PERP: polyethylene-reflected plutonium

sigf: fission cross sections

sigs: scattering cross sections

sigt: total cross sections 